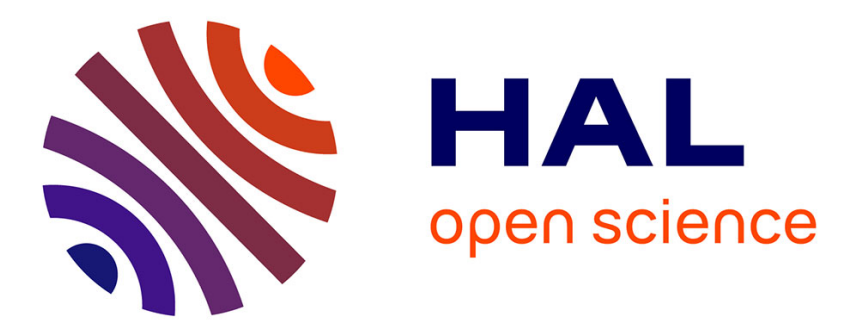

\title{
Characteristics of African easterly waves associated with tropical cyclogenesis in the Cape Verde Islands region in July-August-September of 2004-2008
}

\author{
Joël Arnault, Frank Roux
}

\section{- To cite this version:}

Joël Arnault, Frank Roux. Characteristics of African easterly waves associated with tropical cyclogenesis in the Cape Verde Islands region in July-August-September of 2004-2008. Atmospheric Research, 2011, 100 (1), pp.61-82. 10.1016/j.atmosres.2010.12.028 . hal-00996015

\section{HAL Id: hal-00996015 https://hal.science/hal-00996015}

Submitted on 29 Oct 2020

HAL is a multi-disciplinary open access archive for the deposit and dissemination of scientific research documents, whether they are published or not. The documents may come from teaching and research institutions in France or abroad, or from public or private research centers.
L'archive ouverte pluridisciplinaire HAL, est destinée au dépôt et à la diffusion de documents scientifiques de niveau recherche, publiés ou non, émanant des établissements d'enseignement et de recherche français ou étrangers, des laboratoires publics ou privés. 


\title{
Characteristics of African easterly waves associated with tropical cyclogenesis in the Cape Verde Islands region in July-August-September of 2004-2008
}

\author{
Joël Arnault, Frank Roux * \\ Laboratoire d'Aérologie, (Université de Toulouse and CNRS), 14 avenue Edouard Belin, F-31400 Toulouse, France
}

The most common synoptic-scale disturbances related to cyclogenesis over the tropical north Atlantic Ocean are African easterly waves (AEWs) that originated from the northern African continent. However, most of these waves do not evolve in tropical depressions, storms, or hurricanes. The reasons why only few AEWs develop and the necessary conditions for cyclogenetic evolution are still the subject of intense debate.

Tropical cyclogenesis occurring near the Cape Verde Islands in the eastern tropical Atlantic is investigated here with five seasons (JulyAugust-September of 2004-2008) of European Centre for Medium-Range Weather Forecasts analyses, Meteosat-9 images, and National Hurricane Center (National Oceanic and Atmospheric Administration, National Centers for Environmental Prediction) "best track" archives. The nine named storms that first reached tropical depression intensity east of $30^{\circ} \mathrm{W}$, and two among six which developed between 30 and $40^{\circ} \mathrm{W}$, during these five years evolved from intense AEW troughs, associated with low-level cyclonic circulation, weak mid-level anticyclonic Saharan flow to the east, and deep convection near the center of cyclonic vorticity. The cyclogenetic evolution of three AEW troughs, which verified these conditions but failed to develop into named storms, was probably inhibited by unusually dry environment and strong vertical wind shear. The fate of other AEW troughs, which did not satisfy the necessary conditions, is also discussed.

\section{Introduction}

Tropical cyclogenesis occurs only when several necessary environmental conditions are met (e.g. Gray, 1968; Landsea, 2000): (i) warm ocean waters $\left(>26^{\circ} \mathrm{C}\right)$ throughout at least $50 \mathrm{~m}$ depth to fuel the tropical cyclone heat engine, (ii) an atmosphere potentially unstable to moist convection through which the heat stored in the ocean's upper layers is released, (iii) weak vertical wind shear (less than about $10 \mathrm{~m} \mathrm{~s}^{-1}$ between 850 and $200 \mathrm{hPa}$ ), (iv) a relatively moist midtroposphere to inhibit evaporative cooling and the resulting

\footnotetext{
* Corresponding author. Laboratoire d'Aérologie, Observatoire MidiPyrénées, 14 avenue Edouard Belin, F-31400 Toulouse, France. Tel.: + 33 5613327 52; fax: + 33561332790 .

E-mail address: frank.roux@aero.obs-mip.fr (F. Roux).
}

downward transport of cold air by convective downdrafts, (v) a minimum distance of about $5^{\circ}$ from the equator for the Coriolis force to control gradient wind balance, and (vi) a preexisting disturbance with sufficient vorticity and convergence in the low troposphere and/or an anticyclonic and divergent perturbation in the upper troposphere. However, the sequence of events leading to tropical cyclogenesis remains elusive.

Tropical cyclogenesis can result from interactions between mesoscale convective systems (MCSs) and favourable synoptic conditions (e.g. McBride and Zehr, 1981; Raymond et al., 1998; Rodgers and Fritsch, 2001; Simpson et al., 1997). Mesoscale convective vortices (MCVs) produced in MCSs can merge and increase the storm-scale cyclonic vorticity in the low to mid-levels (e.g. Ritchie and Holland, 1997). Alternatively, low-level convergence and cyclonic vorticity can be 
produced by small-scale areas of deep convection associated with co-located strong vertical vorticity, referred to as vortical hot towers (VHTs) (e.g. Hendricks et al., 2004; Montgomery et al., 2006). Both processes are not contradictory and the progressive merging and axisymmetrization of small-scale vortices induced by VHTs would be favoured in a meso- to large-scale rotating environment (e.g. Van Sang et al., 2008).

These considerations hold for tropical cyclogenesis in general. The present work focuses more precisely on the formation of the so-called "Cape Verde tropical cyclones" over the tropical eastern Atlantic off the West African coast (east of $30^{\circ} \mathrm{W}$ ). These storms are frequently intense and long-lived because they can develop over large areas of warm waters before eventually making landfall and causing major destructions (e.g. Frederic, 1979, Hugo, 1989, Andrew, 1991, Fran, 1996, Bonnie, 1998, Floyd, 1999, Ivan, 2004, ...). Sall et al. (2006) proposed an index for detecting such events, based on a 10-year climatology of atmospheric and oceanic conditions over West Africa and the nearby tropical eastern Atlantic. They noted that the sea surface temperature over the studied area is always warmer than $26{ }^{\circ} \mathrm{C}$ during the boreal summer, and the vertical wind shear is rather low. Hence, these two parameters do not seem to be discriminating factors with respect to the birth of tropical cyclones in this region. Sall et al. (2006) also observed that most Cape Verde tropical cyclones are related to Sahelian MCSs whose cyclogenetic evolution ultimately depends on the synoptic conditions they are embedded in. More precisely, they stated that "the combination between vertical vorticity at $925 \mathrm{hPa}$ (accounting for low level convergence) and potential vorticity at $700 \mathrm{hPa}$ (accounting for instability in midlevel layer) discriminate efficiently between strengthening and weakening evolution offshore near the African coast". Sall et al. (2006) also noted that developing cases are associated with more humidity in the mid-troposphere above the monsoon layer.

African easterly waves (AEWs) are wave-like disturbances that propagate over West Africa and the tropical Atlantic between 10 and $20^{\circ} \mathrm{N}$, with wavelengths of $2000-4000 \mathrm{~km}$, periods of 3-5 days, and maximum amplitude at the African easterly jet (AEJ) level near $700 \mathrm{hPa}$ or $3000 \mathrm{~m}$ altitude (e.g. Reed et al., 1977; Riehl, 1954). A large proportion of Atlantic hurricanes evolve from AEW troughs and the United States' National Hurricane Center (NHC) has been tracking AEWs operationally for decades (e.g. Avila and Clark, 1989). But the detailed processes through which an AEW trough spawns a tropical depression, storm or cyclone, and the reasons why some AEW troughs develop close to the African coast or further west are not well understood. Carlson (1969a) found no evident correlation between the intensity of an AEW trough leaving the West African coast and cyclogenetic evolution over the tropical eastern Atlantic Ocean. However, Thorncroft and Hodges (2001) proposed that the cyclogenetic evolution of AEW troughs depends on their low-level amplitude when they leave the West African coast. Lin et al. (2005) studied the AEW trough which evolved into Hurricane Alberto (2000). They identified three successive convective genesis and lysis periods before the final cyclogenesis evolution occurred off the Guinean coast (Alberto became a tropical depression near $11^{\circ} \mathrm{N}, 18^{\circ} \mathrm{W}$ ). For the same case study, Berry and Thorncroft (2005) suggested that the merging of cyclonic vorticity in the AEW trough and cyclonic vortices of convective origin over the Guinean Highlands favoured this evolution.

Most studies have focused on waves propagating south of the AEJ at the jet level. Carlson (1969b), Burpee (1974), and Reed et al. (1977) observed low-level cyclonic vortices moving westward on the northern flank of the AEJ, at the southern fringe of the Sahara. Northern vortices are associated with dry shallow convection and generally dissipate when they reach the West African coast. However, Pytharoulis and Thorncroft (1998) hypothesized that such northern low-level vortices could be low-level precursors of tropical cyclones forming off the West African coast. A complete tracking history of northerly and southerly vortices by Fink et al. (2004) for all three phases of the Global Atmospheric Research Program Atlantic Tropical Experiment (GATE) revealed that, although northerly and southerly vortices often coexist at least during part of their lifetime, they rarely merge near the west coast of Africa. Likewise, Zawislak and Zipser (2010) concluded that low-level northern disturbances are generally dry and quickly weaken after crossing the West African coast. On the contrary, Ross and Krishnamurti (2007) found that the southern and northern wave regimes can interact, merge and produce a more vigorous trough, instrumental to Atlantic tropical cyclogenesis.

According to Arnault and Roux (2009), the intensification of the "pre-Helene (2006) disturbance" in a tropical depression, south of Cape Verde Islands (near $12^{\circ} \mathrm{N}, 22^{\circ} \mathrm{W}$ ) on 11-12 September 2006, was the result of a geostrophic adjustment through which barotropic conversion of zonal kinetic energy from the AEJ strengthen the cyclonic circulation so as to balance the convectively induced warm anomaly aloft. Arnault and Roux (2010) also showed that the socalled "Perturbation-D" observed on 26-27 September 2006 during AMMA-SOP3 (Third Special Observation Period of the African Monsoon Multidisciplinary Analyses, Redelsperger et al., 2006) failed to develop over the ocean, although its evolution over the continent was relatively similar, owing to a lack of barotropic conversion in the low to mid levels. Likewise, Ross et al. (2009) found that developing AEWs observed in August and September 2006 during NAMMA (the National Administration for Space and Aeronautics' extension of AMMA, Zipser et al., 2009) - including the "preHelene (2006) disturbance" - were characterized by positive barotropic conversion, while non developing ones had negative barotropic conversions. For these AEWs, baroclinic conversion did not play a significant role because positive energy conversion was either small or offset by larger negative conversion.

During the summer season, Carlson and Prospero (1972) observed pulses of warm, dry and dust-laden Saharan air in the mid troposphere over the northern tropical Atlantic Ocean. These Saharan air pulses occur off the West African coast, in relation with AEW ridges and anticyclonic winds. The hot Saharan air layer (SAL) at mid-levels would enhance meridional temperature gradients, strengthen the AEJ, and favour baroclinic growth of AEWs (Karyampudi and Carlson, 1988). Saharan air layer outbreaks could also play a role in the initiation of convection over the ocean during tropical cyclogenesis off West Africa through dynamic, 
thermodynamic and microphysical forcing associated with dust (e.g. Jenkins et al., 2008; Karyampudi et al., 1999). On the other hand, Dunion and Velden (2004) found that SAL has an inhibitive influence on tropical cyclone development through increased stability from the low-level temperature inversion, increased vertical wind shear and dry air intrusion in the mid troposphere. In a case-study of a non-developing AEW trough, Arnault and Roux (2010) showed that the anticyclonic flow associated with the upstream AEW ridge was enhanced by mid-level Saharan anticyclonic flow, stretching the cyclonic vortex in the AEW trough and prohibiting further intensification.

These previous works confirmed that both synoptic and convective processes participate in tropical cyclogenesis from AEW troughs over the tropical eastern Atlantic Ocean off the West African coast. The present study aims at clarifying these conditions through comparing the characteristics of AEW troughs which led or not to "Cape Verde tropical cyclogenesis" during boreal summers of 2004 to 2008. Section 2 describes the 5-year data set used in this work. Phenomena at synoptic and meso-scales are discussed in Sections 3: AEW troughs, 4: mid-level vorticity, 5: low-level vorticity, 6: anticyclonic circulation, and 7: convective processes. A conceptual view of the phenomenon is then proposed in Section 8. Conclusions and some perspectives are finally given in Section 9.

\section{Data}

The large-scale atmospheric conditions associated with Cape Verde tropical cyclogenesis are deduced here from ECMWF (European Centre for Medium-Range Weather Forecasts) operational analyses available in the AMMA data base for July-August-September 2004-2008 (JAS 04-08). It must be outlined that, in 2006, the ECMWF model has been upgraded from a triangular truncation at wavenumber 511 to 799. The special ECMWF reanalyses for summer 2006 including the additional AMMA data (Agusti-Panareda et al., 2009) have not been used in order to keep some homogeneity between the five successive seasons. Air temperature, relative humidity, geopotential heights, wind components, relative vertical vorticity and horizontal divergence are available on a regular latitude-longitude grid of $1.125^{\circ}$ resolution, at 21 pressure levels between 1000 and $1 \mathrm{hPa}$, at 00, 06, 12 and 18 UTC. These data also include daily sea surface temperatures (SSTs).

The convective activity is deduced from the hourly Meteosat-9 (Meteosat-8 in July-August 2004) brightness temperature images in the "water vapour" channel $n^{\circ} 6$ at 6.85-7.85 $\mu \mathrm{m}$. For 2004, 2005 and 2006, these data are available in the AMMA data base. For 2007 and 2008, they were kindly provided by SATMOS (Service d'Archivage et de Traitement Météorologique des Observations Spatiales, Centre National de la Recherche Scientifique and Météo-France). The Meteosat- 9 raw images have been interpolated on a regular latitude-longitude grid of $0.027^{\circ}$ resolution and a brightness temperature resolution of $0.5 \mathrm{~K}$.

The genesis locations of tropical depressions, which became tropical storms and hurricanes, in the tropical Atlantic basin are from the HURricane DATa base (HURDAT or "best-track") maintained by NHC. This dataset was created partially by using subjective interpretations of hurricane forecasters and satellite analysts. Hence, the starting location and time of a tropical depression given by NHC might be somewhat subjective. Detailed information of the genesis of each named storm can be found in Franklin et al. (2006) for the Atlantic Hurricane season of 2004, Beven et al. (2008) for 2005, Franklin and Brown (2008) for 2006, Brennan et al. (2009) for 2007, Brown et al. (2010) for 2008.

It must be outlined that this 5-year data set is too short a sample for statistically sounded conclusions to be drawn. Our goal here is to identify physical processes plausibly associated with the cyclogenetic evolution of AEWs in the Cape Verde Islands region. The verification of their applicability for forecasting would deserve a more comprehensive study with a longer (decade or more) investigation period with far more AEWs. This is however out of the scope of the present paper.

\section{AEW troughs crossing the West African coast and named storms}

\subsection{Detection of AEW troughs}

The aim of this work is to investigate the relative contributions of synoptic and convective processes in the cyclogenetic or non-cyclogenetic evolution of AEW troughs observed off the West African coast in JAS 04-08. Firstly, the AEW troughs passing over the Cape Verde Islands region must be objectively detected in the ECMWF analyses. Berry et al. (2006) proposed a technique for automatically tracking AEW troughs axes over West Africa and the nearby Atlantic Ocean. However, this method identifies multiple troughs, some of which can be associated with mesoscale and shortlived maxima of convective origin, not only with large-scale and long-lived AEW troughs (Kerns et al., 2008).

Here, AEW troughs are identified by relative maxima of the area with relative vorticity at $700 \mathrm{hPa}$ larger than a given threshold in domain $\Omega\left(8-22^{\circ} \mathrm{N}, 20-28^{\circ} \mathrm{W}\right)$ centered on Cape Verde Islands. This domain is centered over the Cape Verde Islands region, its meridional extension is such that all AEW troughs can be detected whatever their latitude and its longitudinal extension is such that AEW troughs can be detected before tropical cyclogenesis occurred. Fig. 1 shows the evolution of this criterion for JAS 2004 using three different relative vorticity thresholds $(+2.5,+5$, and $\left.+7.5 \times 10^{-5} \mathrm{~s}^{-1}\right)$. About thirty AEW troughs are detected in JAS 2004 and this number does not vary much with the threshold value $(31,29$, and 30 for thresholds $+2.5,+5$, and $+7.5 \times 10^{-5} \mathrm{~s}^{-1}$, respectively). Cyclonic vorticity values larger than $+5 \times 10^{-5} \mathrm{~s}^{-1}$ at $700 \mathrm{hPa}$ represent the last decile (highest $10 \%$ ) of all the cyclonic vorticity values in domain $\Omega$ for JAS $04-08$. In the following, this value is chosen as the relative vorticity threshold to identify AEW troughs.

The period of approximately three days between each trough is in agreement with previous studies. The zonal propagation speed derived from the successive positions of vorticity centers of AEW troughs in domain $\Omega$ (deduced here as the weighted mean of vorticity values larger than $+5 \times 10^{-5} \mathrm{~s}^{-1}$ at $700 \mathrm{hPa}$ ) is $6.3 \mathrm{~m} \mathrm{~s}^{-1}$ with a standard deviation of $1.5 \mathrm{~m} \mathrm{~s}^{-1}$. This is relatively slow, but compatible with previous estimates of $5-10 \mathrm{~m} \mathrm{~s}^{-1}$ or $4-8^{\circ}$ longitude per 

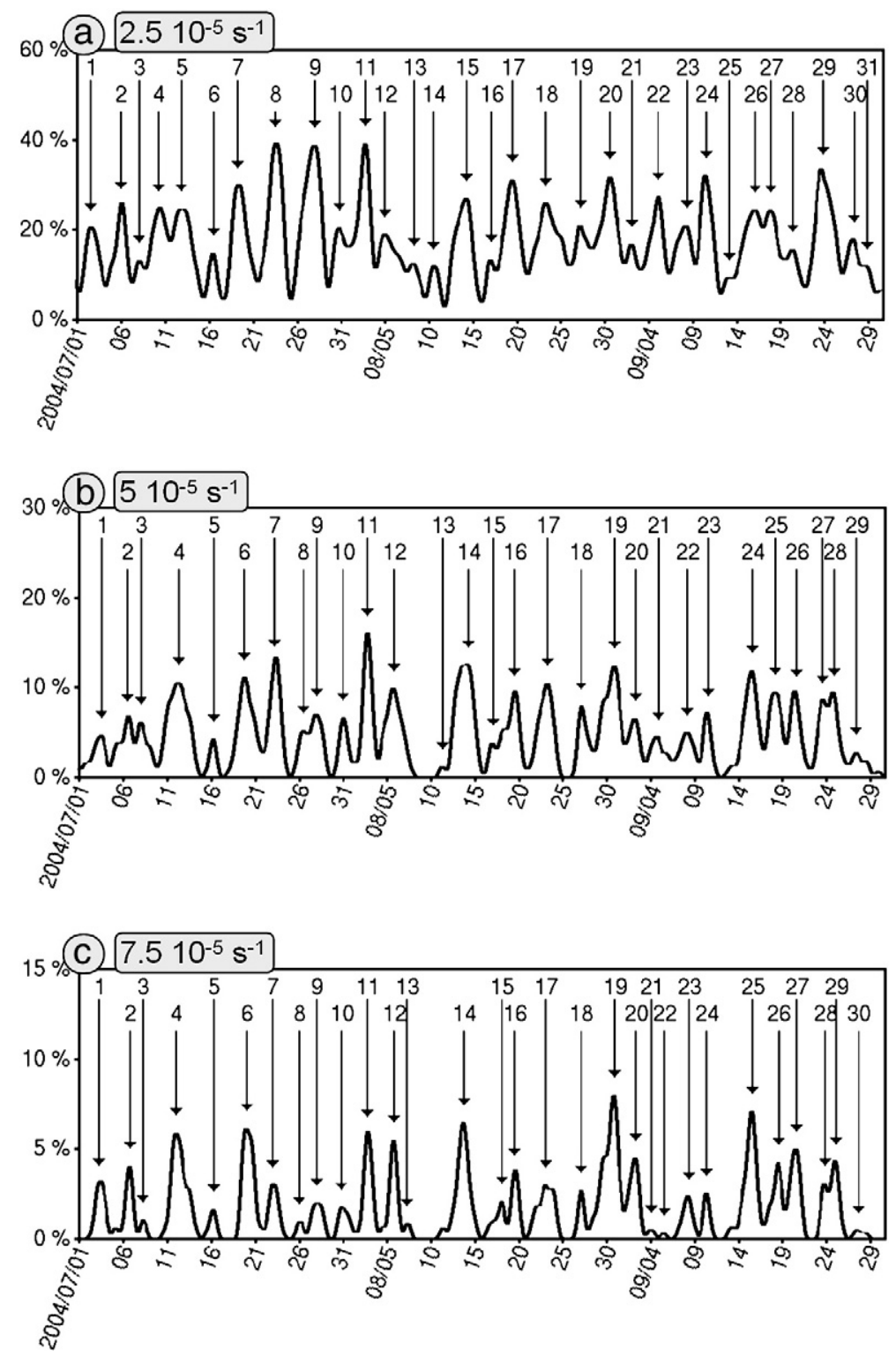

Fig. 1. (a) evolution of the relative area of vorticity higher than $+2.5 \times 10^{-5} \mathrm{~s}^{-1}$ in the domain $\Omega\left(8-22^{\circ} \mathrm{N}, 20-28^{\circ} \mathrm{W}\right)$ at pressure level $700 \mathrm{hPa}$ from $00 \mathrm{UTC} 1$ July 2004 until 00 UTC 1 October 2004 UTC (scale on the vertical axis). The horizontal axis gives the time in days. Maxima in this curve have been associated with AEW troughs that have been numbered; (b) as in (a), except with a vorticity threshold of $+5 \times 10^{-5} \mathrm{~s}^{-1}$; (c) as in (a), except with a vorticity threshold of $+7.5 \times 10^{-5} \mathrm{~s}^{-1}$.

day for the propagation speed of AEWs (e.g. Berry et al., 2006).

Fig. 2 shows six AEW troughs detected with this threshold in the Cape Verde Islands region in 2004. The horizontal distribution of cyclonic vorticity is not uniform and the latitudinal position of AEW troughs is quite variable. The meridional limits of domain $\Omega\left(8^{\circ}\right.$ and $\left.22^{\circ} \mathrm{N}\right)$ are chosen so that all the AEW troughs crossing the West African coast are detected. This domain has also to be narrow enough in longitude to avoid the simultaneous presence of two AEW troughs. The $8^{\circ}$ zonal width $\left(20-28^{\circ} \mathrm{W}\right)$ is much smaller than the $20-40^{\circ}$ wavelength of AEWs, which generally prevents such a situation to occur.
With this criterion, 28, 32, 25, 27, and 30 AEW troughs are detected for JAS of 2004, 2005, 2006, 2007, and 2008, respectively (Tables $1-5$ ). In few cases, successive relative maxima of area with vertical vorticity at $700 \mathrm{hPa}$ larger than $5 \times 10^{-3} \mathrm{~s}^{-1}$, separated by less than $24 \mathrm{~h}$, have been associated with single AEW troughs ( $\mathrm{n}^{\circ} 27 \mathrm{a}$, b in 2004; 13a,b, 14a,b, 18a,b, and 32a,b in 2005; 4a,b and 23a,b in 2006; 4a,b, 19a,b, 22a,b in 2007; 13a,b and 26a,b in 2008; see Tables 1-5). The averaged circulation at $700 \mathrm{hPa}$ for these $142 \mathrm{AEW}$ troughs, relative to their respective vorticity centers, (Fig. 3a) displays cyclonically curved winds extending approximately $3^{\circ}$ longitude northward and $7^{\circ}$ southward, westward and eastward, but the circulation is not closed. 

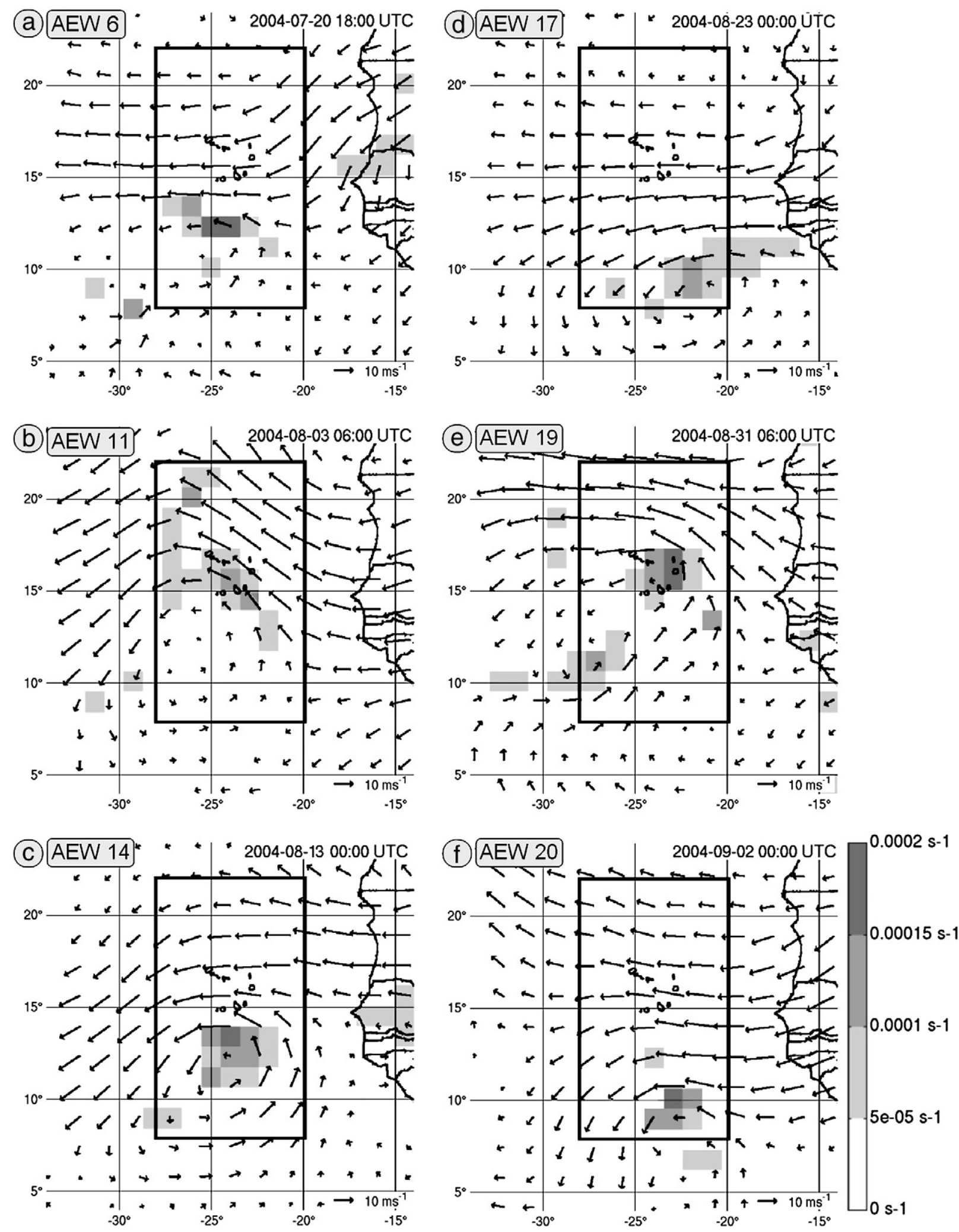

Fig. 2. (a) horizontal winds (arrows) and relative vorticity $\left(\mathrm{s}^{-1}\right.$ ) at $700 \mathrm{hPa}$ when AEW trough $\mathrm{n}^{\circ} 6$ of 2004 crossed domain $\Omega$ (black rectangle); (b, c, d, e, and f) as in (a), except for AEW troughs $n^{\circ} 11,14,17,19$ and 20 of 2004 respectively. Vorticity scale is indicated in the bottom right. The scale for horizontal winds is indicated in the bottom right of each panel. 
Table 1

Characteristics of AEW troughs in JAS 2004 (see the text for details) and associated tropical cyclogenesis events [the date and longitude of the named storms correspond to the first time and location where they reach Tropical Storm status (10-min averaged winds $\left.>17 \mathrm{~m} \mathrm{~s}^{-1}\right)$ ]. Light and dark shading denotes "intense AEW troughs with favourable synoptic conditions and deep convective activity" that did and did not develop, respectively, into named storms. In the lower right, the number of storms developing from those AEW troughs is indicated in parentheses.

\begin{tabular}{|c|c|c|c|c|c|c|c|c|c|}
\hline \multicolumn{2}{|l|}{2004} & \multicolumn{4}{|c|}{ AEW characteristics } & \multicolumn{4}{|c|}{ Tropical cyclogenesis } \\
\hline AEW \# & Date in $\Omega$ & \begin{tabular}{|l|} 
Intense \\
cyclonic \\
circulation at \\
$700 \mathrm{hPa}$
\end{tabular} & \begin{tabular}{|l|} 
Cyclonic \\
circulation at \\
$1000 \mathrm{hPa}$
\end{tabular} & $\begin{array}{l}\text { Anticyclonic } \\
\text { circulation at } \\
700 \mathrm{hpa}\end{array}$ & $\begin{array}{l}\text { Deep } \\
\text { convection }\end{array}$ & $<30^{\circ} \mathrm{W}$ & $30-40^{\circ} \mathrm{W}$ & $40-60^{\circ} \mathrm{W}$ & $>60^{\circ} \mathrm{W}$ \\
\hline 1 & $03 \mathrm{Jul}$ & - & & & & & & & \\
\hline 2 & $06 \mathrm{Jul}$ & & & & & & & & \\
\hline 3 & $08 \mathrm{Jul}$ & & & & & & & & \\
\hline 4 & $13 \mathrm{Jul}$ & - & & & $\bullet$ & & & & \\
\hline 5 & $16 \mathrm{Jul}$ & & & & & & & & \\
\hline 6 & $20 \mathrm{Jul}$ & - & $\bullet$ & & & & & & \\
\hline 7 & $23 \mathrm{Jul}$ & & & - & & & & & \\
\hline 8 & $26 \mathrm{Jul}$ & & & & & & & & \\
\hline 9 & $28 \mathrm{Jul}$ & - & & & & & & & \\
\hline 10 & $31 \mathrm{Jul}$ & & & & & & & $\begin{array}{l}\text { Bonnie } \\
03 \text { Aug }\end{array}$ & \\
\hline 11 & 03 Aug & & & - & & & & & \\
\hline 12 & 06 Aug & $\bullet$ & & & & & & $\begin{array}{l}\text { Charley } \\
09 \text { Aug }\end{array}$ & \\
\hline 13 & 11 Aug & & & & & & & $\begin{array}{l}\text { Earl } \\
13 \text { Aug }\end{array}$ & \\
\hline 14 & 13 Aug & - & - & & - & $\begin{array}{l}\text { Danielle } \\
13 \text { Aug }\end{array}$ & & & \\
\hline 15 & 17 Aug & & & - & & & & & \\
\hline 16 & 20 Aug & $\bullet$ & & & & & & & \\
\hline 17 & 23 Aug & - & $\bullet$ & & & & $\begin{array}{l}\text { Frances } \\
25 \text { Aug }\end{array}$ & & \\
\hline 18 & 27 Aug & & & - & & & & & \\
\hline 19 & 31 Aug & - & $\bullet$ & & $\bullet$ & $\begin{array}{l}\text { Ivan } \\
12 \text { Sep }\end{array}$ & & & \\
\hline 20 & $02 \mathrm{Sep}$ & - & - & & - & & & & \\
\hline 21 & 05 Sep & & & $\bullet$ & & & & & \\
\hline 22 & 08 Sep & & & & & & & $\begin{array}{l}\text { Jeanne } \\
13 \text { Sep }\end{array}$ & \\
\hline 23 & $10 \mathrm{Sep}$ & & & - & & & & & \\
\hline 24 & 15 Sep & - & $\bullet$ & & - & $\begin{array}{l}\text { Karl } \\
16 \text { Sep } \\
\end{array}$ & & & \\
\hline 25 & $18 \mathrm{Sep}$ & $\bullet$ & $\bullet$ & & - & & $\begin{array}{l}\text { Lisa } \\
19 \text { Sep }\end{array}$ & & \\
\hline 26 & $20 \mathrm{Sep}$ & - & & & - & & & & $\begin{array}{l}\text { Matthew } \\
08 \text { Oct }\end{array}$ \\
\hline $\begin{array}{l}27 \mathrm{a} \\
27 \mathrm{~b}\end{array}$ & $\begin{array}{l}23 \mathrm{Sep} \\
24 \mathrm{Sep}\end{array}$ & - & & - & $\bullet$ & & & & \\
\hline 28 & $27 \mathrm{Sep}$ & & & & & & & & \\
\hline \multicolumn{2}{|c|}{2004 AEW trough } & \begin{tabular}{|l|} 
Intense AEW \\
troughs
\end{tabular} & $\begin{array}{l}\text { Intense AEW } \\
\text { troughs with } \\
\text { low-level } \\
\text { cyclonic } \\
\text { circulation }\end{array}$ & $\begin{array}{l}\text { Intense AEW } \\
\text { troughs with } \\
\text { favourable } \\
\text { synotic } \\
\text { conditions }\end{array}$ & $\begin{array}{l}\text { Intense AEW } \\
\text { troughs with } \\
\text { favourable } \\
\text { synotic and } \\
\text { convective } \\
\text { conditions }\end{array}$ & $<30^{\circ} \mathrm{W}$ & $30-40^{\circ} \mathrm{W}$ & $40-60^{\circ} \mathrm{W}$ & $>60^{\circ} \mathrm{W}$ \\
\hline \multicolumn{2}{|l|}{28} & 14 & 7 & 7 & 5 & $3(3)$ & $2(1)$ & $4(0)$ & $4(1)$ \\
\hline
\end{tabular}


Table 2

As in Table 1, except for 2005.

\begin{tabular}{|c|c|c|c|c|c|c|c|c|c|}
\hline \multicolumn{2}{|l|}{2005} & \multicolumn{4}{|c|}{ AEW characteristics } & \multicolumn{4}{|c|}{ Tropical cyclogenesis } \\
\hline AEW \# & Date in $\Omega$ & \begin{tabular}{|l} 
Intense \\
cyclonic \\
circulation at \\
$700 \mathrm{hPa}$
\end{tabular} & $\begin{array}{l}\text { Cyclonic } \\
\text { circulation at } \\
1000 \mathrm{hPa}\end{array}$ & $\begin{array}{l}\text { Anticyclonic } \\
\text { circulation at } \\
700 \mathrm{hPa}\end{array}$ & $\begin{array}{l}\text { Deep } \\
\text { convection }\end{array}$ & $<30^{\circ} \mathrm{W}$ & $30-40^{\circ} \mathrm{W}$ & $40-60^{\circ} \mathrm{W}$ & $>60^{\circ} \mathrm{W}$ \\
\hline 1 & $02 \mathrm{Jul}$ & & & & & & & & \\
\hline 2 & $04 \mathrm{Jul}$ & & & & & & & & \\
\hline 3 & $08 \mathrm{Jul}$ & & & & $\bullet$ & & & Emily $11 \mathrm{Jul}$ & \\
\hline 4 & $11 \mathrm{Jul}$ & $\bullet$ & & & - & & & & $\begin{array}{l}\text { Franklin } 21 \text { Jul } \\
\text { Gert } 23 \text { Jul }\end{array}$ \\
\hline 5 & $15 \mathrm{Jul}$ & & & & & & & & \\
\hline 6 & $17 \mathrm{Jul}$ & & & & & & & & \\
\hline 7 & $19 \mathrm{Jul}$ & & & & & & & & \\
\hline 8 & $21 \mathrm{Jul}$ & & & & & & & & \\
\hline 9 & $25 \mathrm{Jul}$ & & & & $\bullet$ & & & & $\begin{array}{l}\text { Harvey } \\
02 \text { Aug }\end{array}$ \\
\hline 10 & $26 \mathrm{Jul}$ & & & & & & & & \\
\hline 11 & $28 \mathrm{Jul}$ & & & & & & & & \\
\hline 12 & $31 \mathrm{Jul}$ & & & & & & & & \\
\hline $\begin{array}{l}13 a \\
13 b\end{array}$ & $\begin{array}{l}02 \text { Aug } \\
03 \text { Aug }\end{array}$ & & & & $\bullet$ & & $\begin{array}{l}\text { Irene } \\
04 \text { Aug }\end{array}$ & & \\
\hline $\begin{array}{l}14 a \\
14 b\end{array}$ & $\begin{array}{l}07 \text { Aug } \\
08 \text { Aug }\end{array}$ & & & & & & & & \\
\hline 15 & 10 Aug & & & & & & & & Jose 22 Aug \\
\hline 16 & 12 Aug & & & & & & & & \\
\hline 17 & 14 Aug & & & $\bullet$ & & & & & \\
\hline $\begin{array}{l}18 \mathrm{a} \\
18 \mathrm{~b}\end{array}$ & $\begin{array}{l}16 \text { Aug } \\
17 \text { Aug }\end{array}$ & & & & & & & & \\
\hline 19 & 19 Aug & & & $\bullet$ & & & & & \\
\hline 20 & 22 Aug & - & & $\bullet$ & $\bullet$ & & & & \\
\hline 21 & 26 Aug & & & & & & & Lee 28 Aug & \\
\hline 22 & 28 Aug & & & & & & & $\begin{array}{l}\text { Maria } \\
01 \text { Sep }\end{array}$ & \\
\hline 23 & 31 Aug & $\bullet$ & & & & & & & Nate 05 Sep \\
\hline 24 & 03 Sep & & & & & & & & \\
\hline 25 & 05 Sep & & & & & & & & \\
\hline 26 & 08 Sep & & & & & & & & Rita 18 Sep \\
\hline 27 & 10 Sep & & & & & & & $\begin{array}{l}\text { Philippe } \\
17 \text { Sep }\end{array}$ & \\
\hline 28 & 12 Sep & & & & & & & & \\
\hline 29 & 14 Sep & & & $\bullet$ & & & & & \\
\hline 30 & 19 Sep & & & & & & & & \\
\hline 31 & 23 Sep & & & & $\bullet$ & & & & Stan 01 Oct \\
\hline $\begin{array}{l}32 \mathrm{a} \\
32 \mathrm{a}\end{array}$ & $\begin{array}{l}27 \text { Sep } \\
28 \text { Sep }\end{array}$ & • & & & $\bullet$ & & & & $\begin{array}{l}\text { Tammy } \\
05 \text { Sep }\end{array}$ \\
\hline \multicolumn{2}{|c|}{2005 AEW troughs } & $\begin{array}{l}\text { Intense AEW } \\
\text { troughs }\end{array}$ & $\begin{array}{l}\text { Intense AEW } \\
\text { troughs with } \\
\text { low-level } \\
\text { cyclonic } \\
\text { circulation }\end{array}$ & $\begin{array}{l}\text { Intense AEW } \\
\text { troughs with } \\
\text { favourable } \\
\text { synoptic } \\
\text { conditions }\end{array}$ & $\begin{array}{l}\text { Intense AEW } \\
\text { troughs with } \\
\text { favourable } \\
\text { synoptic and } \\
\text { convective } \\
\text { conditions }\end{array}$ & $<30^{\circ} \mathrm{W}$ & $30-40^{\circ} \mathrm{W}$ & $40-60^{\circ} \mathrm{W}$ & $>60^{\circ} \mathrm{W}$ \\
\hline \multicolumn{2}{|l|}{32} & 4 & 0 & 0 & 0 & $0(0)$ & $1(0)$ & $4(0)$ & $1(0)$ \\
\hline
\end{tabular}


Table 3

As in Table 1, except for 2006.

\begin{tabular}{|c|c|}
\hline \multicolumn{2}{|l|}{2006} \\
\hline AEW \# & Date in $\Omega$ \\
\hline 1 & $04 \mathrm{Jul}$ \\
\hline 2 & $08 \mathrm{Jul}$ \\
\hline 3 & $10 \mathrm{Jul}$ \\
\hline $\begin{array}{l}4 a \\
4 b\end{array}$ & $\begin{array}{l}12 \mathrm{Jul} \\
13 \mathrm{Jul}\end{array}$ \\
\hline 5 & $16 \mathrm{Jul}$ \\
\hline 6 & $18 \mathrm{Jul}$ \\
\hline 7 & $21 \mathrm{Jul}$ \\
\hline 8 & $25 \mathrm{Jul}$ \\
\hline 9 & $27 \mathrm{Jul}$ \\
\hline 10 & $30 \mathrm{Jul}$ \\
\hline 11 & 01 Aug \\
\hline 12 & 05 Aug \\
\hline 13 & 07 Aug \\
\hline 14 & 14 Aug \\
\hline 15 & 16 Aug \\
\hline 16 & 21 Aug \\
\hline 17 & 26 Aug \\
\hline 18 & 01 Sep \\
\hline 19 & 09 Sep \\
\hline 20 & 12 Sep \\
\hline 21 & 17 Sep \\
\hline 22 & 19 Sep \\
\hline $\begin{array}{l}23 a \\
23 b\end{array}$ & $\begin{array}{l}22 \text { Sep } \\
23 \text { Sep }\end{array}$ \\
\hline 24 & 25 Sep \\
\hline 25 & 28 Sep \\
\hline
\end{tabular}

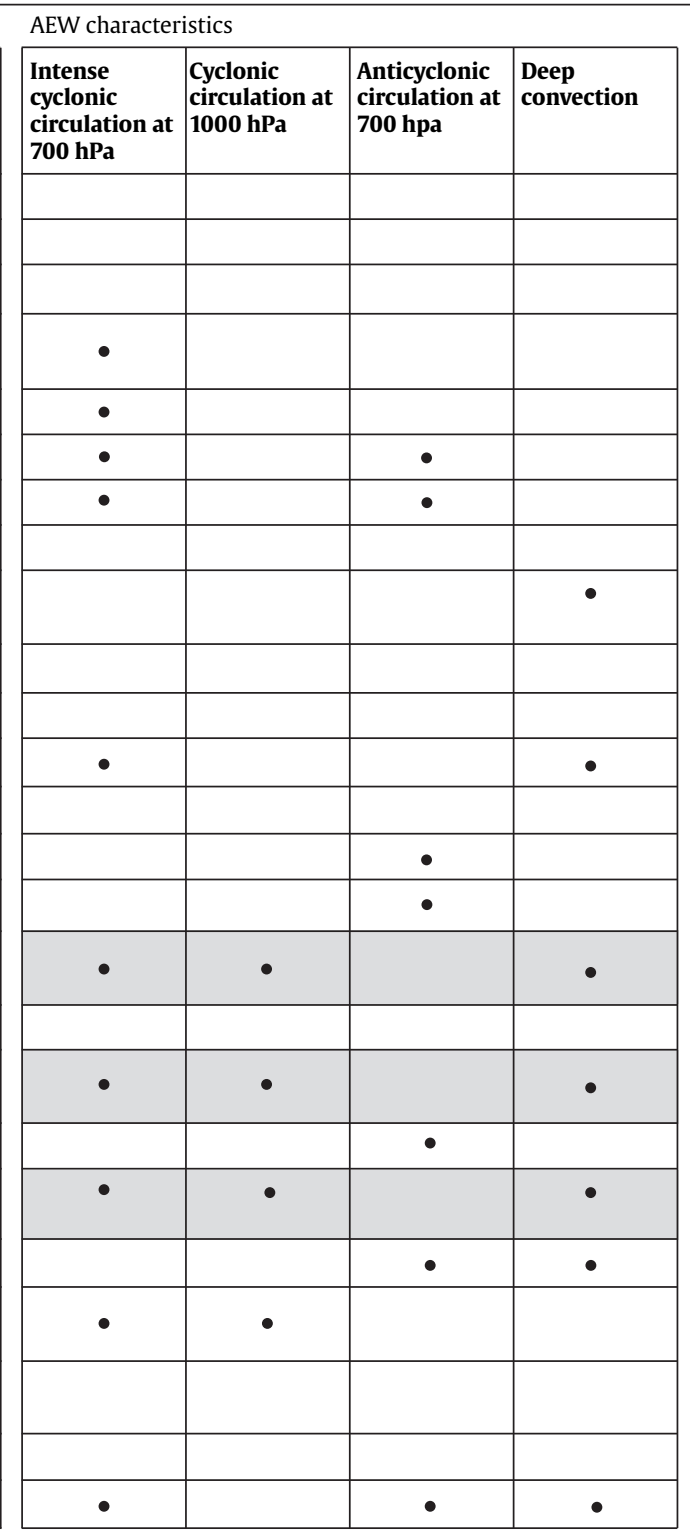

Tropical cyclogenesis
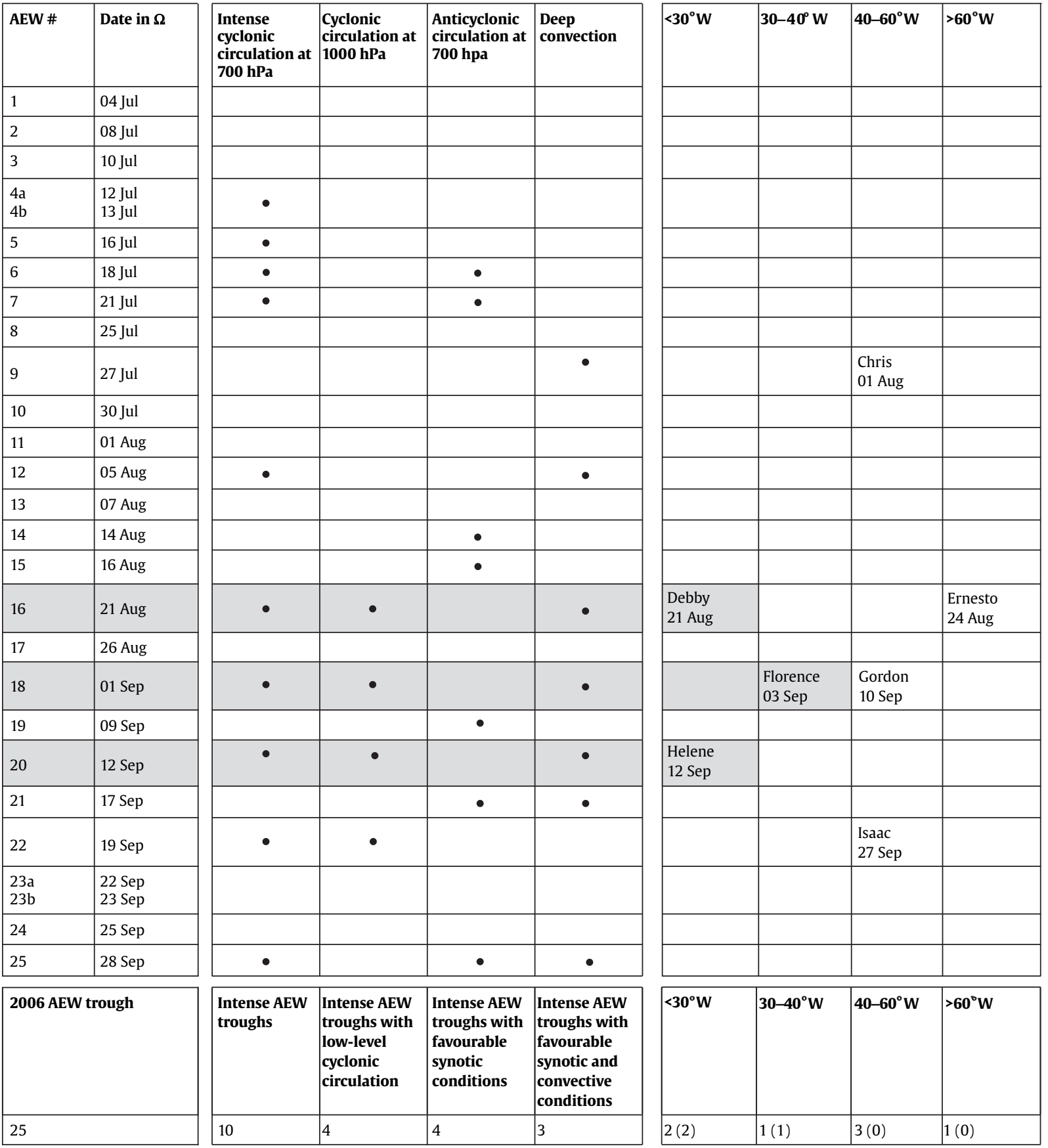

\subsection{Detection of intense AEW troughs}

The intensity of AEW troughs in the Cape Verde Islands region certainly plays an important role in tropical cyclogenesis, as it favours convective development (e.g. Davis and Trier, 2007) and facilitates the up-scale organization of convective-scale cyclonic vortices (e.g. Berry and Thorncroft, 2005; Van Sang et al., 2008). We identify these intense AEW troughs by the presence of relative vorticity values at $700 \mathrm{hPa}$ larger than $10^{-4} \mathrm{~s}^{-1}$ in domain $\Omega$. This value is close to the upper percentile (highest $1 \%$ ) of the vorticity values for the whole data set. The verification of this criterion during more than $24 \mathrm{~h}$ defines an "intense AEW trough".

In JAS of 2004, 2005, 2006, 2007 and 2008, 14, 4, 10, 11 and 15 intense AEW troughs occurred, respectively (Tables 1-5). The averaged circulation at $700 \mathrm{hPa}$, relative to their 
Table 4

As in Table 1, except for 2007.

\begin{tabular}{|c|c|c|c|c|c|c|c|c|c|}
\hline \multicolumn{2}{|l|}{2007} & \multicolumn{4}{|c|}{ AEW characteristics } & \multicolumn{4}{|c|}{ Tropical cyclogenesis } \\
\hline AEW \# & Date in $\Omega$ & $\begin{array}{l}\text { Intense } \\
\text { cyclonic } \\
\text { circulation at } \\
700 \mathrm{hPa}\end{array}$ & $\begin{array}{l}\text { Cyclonic } \\
\text { circulation at } \\
1000 \mathrm{hPa}\end{array}$ & $\begin{array}{l}\text { Anticyclonic } \\
\text { circulation at } \\
700 \mathrm{hPa}\end{array}$ & $\begin{array}{l}\text { Deep } \\
\text { convection }\end{array}$ & $<30^{\circ} \mathrm{W}$ & $30-40^{\circ} \mathrm{W}$ & $40-60^{\circ} \mathrm{W}$ & $>60^{\circ} \mathrm{W}$ \\
\hline 1 & $03 \mathrm{Jul}$ & - & & & & & & & \\
\hline 2 & $05 \mathrm{Jul}$ & & & & & & & & \\
\hline 3 & $10 \mathrm{Jul}$ & & & & & & & & \\
\hline $4 a$ & $13 \mathrm{Jul}$ & & & & & & & & \\
\hline $4 \mathrm{~b}$ & $14 \mathrm{Jul}$ & & & & $\bullet$ & & & & \\
\hline 5 & $17 \mathrm{Jul}$ & $\bullet$ & & & & & & & \\
\hline 6 & $19 \mathrm{Jul}$ & & & & & & & & \\
\hline 7 & $23 \mathrm{Jul}$ & & & & $\bullet$ & & & & \\
\hline 8 & $26 \mathrm{Jul}$ & $\bullet$ & & & - & & & & \\
\hline 9 & $29 \mathrm{Jul}$ & - & & & $\bullet$ & & & & \\
\hline 10 & 02 Aug & $\bullet$ & & & & & & & \\
\hline 11 & 04 Aug & & $\bullet$ & - & & & & & $\begin{array}{l}\text { Erin } \\
15 \text { Aug }\end{array}$ \\
\hline 12 & 06 Aug & & & & - & & & & \\
\hline 13 & 10 Aug & & & - & & & & & \\
\hline 14 & 12 Aug & $\bullet$ & $\bullet$ & & $\bullet$ & $\begin{array}{l}\text { Dean } \\
13 \text { Aug }\end{array}$ & & & \\
\hline 15 & 14 Aug & $\bullet$ & & & & & & & \\
\hline 16 & 17 Aug & & & & & & & & \\
\hline 17 & 23 Aug & - & - & & - & & & & \\
\hline 18 & 26 Aug & & & & & & & $\begin{array}{l}\text { Felix } \\
31 \text { Aug }\end{array}$ & \\
\hline $\begin{array}{l}19 a \\
19 b\end{array}$ & $\begin{array}{l}29 \text { Aug } \\
30 \text { Aug }\end{array}$ & & & & & & & & \\
\hline 20 & 02 Sep & & & $\bullet$ & & & & & \\
\hline 21 & 08 Sep & - & & & - & & & $\begin{array}{l}\text { Ingrid } \\
12 \text { Sep }\end{array}$ & \\
\hline $\begin{array}{l}22 a \\
22 b\end{array}$ & $\begin{array}{l}12 \text { Sep } \\
13 \text { Sep }\end{array}$ & & & & & & & & $\begin{array}{l}\text { Lorenzo } \\
25 \text { Sep }\end{array}$ \\
\hline 23 & 15 Sep & & & & & & & & \\
\hline 24 & 18 Sep & & & & & & & & \\
\hline 25 & 21 Sep & & & $\bullet$ & & & & & \\
\hline 26 & 23 Sep & - & & & - & & $\begin{array}{l}\text { Karen } \\
29 \text { Sep }\end{array}$ & & \\
\hline 27 & 27 Sep & - & - & & $\bullet$ & $\begin{array}{l}\text { Melissa } \\
28 \text { Sep }\end{array}$ & & & \\
\hline \multicolumn{2}{|c|}{2007 AEW troughs } & $\begin{array}{l}\text { Intense AEW } \\
\text { troughs }\end{array}$ & $\begin{array}{l}\text { Intense AEW } \\
\text { troughs with } \\
\text { low-level } \\
\text { cyclonic } \\
\text { circulation }\end{array}$ & $\begin{array}{l}\text { Intense AEW } \\
\text { troughs with } \\
\text { favourable } \\
\text { synoptic } \\
\text { conditions }\end{array}$ & $\begin{array}{l}\text { Intense AEW } \\
\text { troughs with } \\
\text { favourable } \\
\text { synoptic and } \\
\text { convective } \\
\text { conditions }\end{array}$ & $<30^{\circ} \mathrm{W}$ & $30-40^{\circ} \mathrm{W}$ & $40-60^{\circ} \mathrm{W}$ & $>60^{\circ} \mathrm{W}$ \\
\hline \multicolumn{2}{|l|}{27} & 11 & 3 & 3 & 3 & $2(2)$ & $1(0)$ & $2(0)$ & $2(0)$ \\
\hline
\end{tabular}

respective vorticity centers, for the 54 intense AEW troughs detected for JAS 04-08 (Fig. 3b) is comparable to that of the 142 AEW troughs (Fig. 3a), but the cyclonic curvature of the easterlies is more pronounced and the circulation is closed.

\subsection{Link with tropical cyclogenesis}

The NHC best-track data set allows to identify the AEW troughs which evolved into tropical depressions (organized 
Table 5

As in Table 1, except for 2008.

\begin{tabular}{|c|c|c|c|c|c|c|c|c|c|}
\hline \multicolumn{2}{|l|}{2008} & \multicolumn{4}{|c|}{ AEW characteristics } & \multicolumn{4}{|c|}{ Tropical cyclogenesis } \\
\hline AEW \# & Date in $\Omega$ & $\begin{array}{l}\text { Intense } \\
\text { cyclonic } \\
\text { circulation at } \\
700 \mathrm{hPa}\end{array}$ & $\begin{array}{l}\text { Cyclonic } \\
\text { circulation at } \\
1000 \mathrm{hPa}\end{array}$ & $\begin{array}{l}\text { Anticyclonic } \\
\text { circulation at } \\
700 \mathrm{hPa}\end{array}$ & $\begin{array}{l}\text { Deep } \\
\text { convection }\end{array}$ & $<30^{\circ} \mathrm{W}$ & $30-40^{\circ} \mathrm{W}$ & $40-60^{\circ} \mathrm{W}$ & $>60^{\circ} \mathrm{W}$ \\
\hline 1 & $03 \mathrm{Jul}$ & $\bullet$ & - & & $\bullet$ & $\begin{array}{l}\text { Bertha } \\
03 \text { Jul }\end{array}$ & & & \\
\hline 2 & $06 \mathrm{Jul}$ & & & & & & & & \\
\hline 3 & $08 \mathrm{Jul}$ & & & & & & & & \\
\hline 4 & $12 \mathrm{Jul}$ & & & & & & & & $\begin{array}{l}\text { Dolly } \\
20 \mathrm{Jul}\end{array}$ \\
\hline 5 & $15 \mathrm{Jul}$ & & & $\bullet$ & & & & & \\
\hline 6 & $18 \mathrm{Jul}$ & & & & & & & & \\
\hline 7 & $23 \mathrm{Jul}$ & $\bullet$ & & & & & & & \\
\hline 8 & $26 \mathrm{Jul}$ & $\bullet$ & & & & & & & \\
\hline 9 & $28 \mathrm{Jul}$ & & & & & & & & \\
\hline 10 & $31 \mathrm{Jul}$ & $\bullet$ & $\bullet$ & $\bullet$ & - & & & & \\
\hline 11 & 05 Aug & $\bullet$ & & & $\bullet$ & & & & \\
\hline 12 & 08 Aug & $\bullet$ & & & & & & & $\begin{array}{l}\text { Fay } \\
15 \text { Aug }\end{array}$ \\
\hline $\begin{array}{l}13 a \\
13 b \\
\end{array}$ & $\begin{array}{l}11 \text { Aug } \\
12 \text { Aug } \\
\end{array}$ & $\bullet$ & $\bullet$ & & $\bullet$ & & & & \\
\hline $14 a$ & 15 Aug & - & - & $\bullet$ & - & & & & $\begin{array}{l}\text { Gustav } \\
25 \text { Aug }\end{array}$ \\
\hline 15 & 20 Aug & $\bullet$ & & & & & & $\begin{array}{l}\text { Hanna } \\
28 \text { Aug }\end{array}$ & \\
\hline 16 & 23 Aug & $\bullet$ & & & & & & & \\
\hline 17 & 26 Aug & & & & $\bullet$ & & & & \\
\hline 18 & 30 Aug & $\bullet$ & • & - & - & & $\begin{array}{l}\text { Ike } \\
01 \text { Sep }\end{array}$ & & \\
\hline 19 & 02 Sep & - & - & & - & $\begin{array}{l}\text { Josephine } \\
\text { 02 Sep }\end{array}$ & & & \\
\hline 20 & 06 Sep & & & & & & & & \\
\hline 21 & 07 Sep & & & 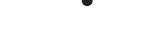 & & & & & \\
\hline 22 & 09 Sep & & & & & & & & \\
\hline 23 & 12 Sep & & & & & & & & \\
\hline 24 & 14 Sep & & & & & & & & $\begin{array}{l}\text { Kyle } \\
25 \text { Sep }\end{array}$ \\
\hline 25 & 16 Sep & & & $\bullet$ & & & & & \\
\hline $\begin{array}{l}26 \mathrm{a} \\
26 \mathrm{~b}\end{array}$ & $\begin{array}{l}18 \text { Sep } \\
19 \text { Sep }\end{array}$ & - & & - & & & & & \\
\hline 27 & 21 Sep & & & $\bullet$ & & & & & \\
\hline 28 & 24 Sep & & & $\bullet$ & & & & & \\
\hline 29 & 27 Sep & & & $\bullet$ & & & & & \\
\hline 30 & 29 Sep & $\bullet$ & & & & & & & \\
\hline \multicolumn{2}{|c|}{2008 AEW troughs } & $\begin{array}{l}\text { Intense AEW } \\
\text { troughs }\end{array}$ & $\begin{array}{l}\text { Intense AEW } \\
\text { troughs with } \\
\text { low-level } \\
\text { cyclonic } \\
\text { circulation }\end{array}$ & $\begin{array}{l}\text { Intense AEW } \\
\text { troughs with } \\
\text { favourable } \\
\text { synoptic } \\
\text { conditions }\end{array}$ & \begin{tabular}{|l|} 
Intense AEW \\
troughs with \\
favourable \\
synoptic and \\
convective \\
conditions \\
\end{tabular} & $<30^{\circ} \mathrm{W}$ & $30-40^{\circ} \mathrm{W}$ & $40-60^{\circ} \mathrm{W}$ & $>60^{\circ} \mathrm{W}$ \\
\hline \multicolumn{2}{|l|}{30} & 15 & 6 & 3 & 3 & $2(2)$ & $1(0)$ & $1(0)$ & $4(0)$ \\
\hline
\end{tabular}



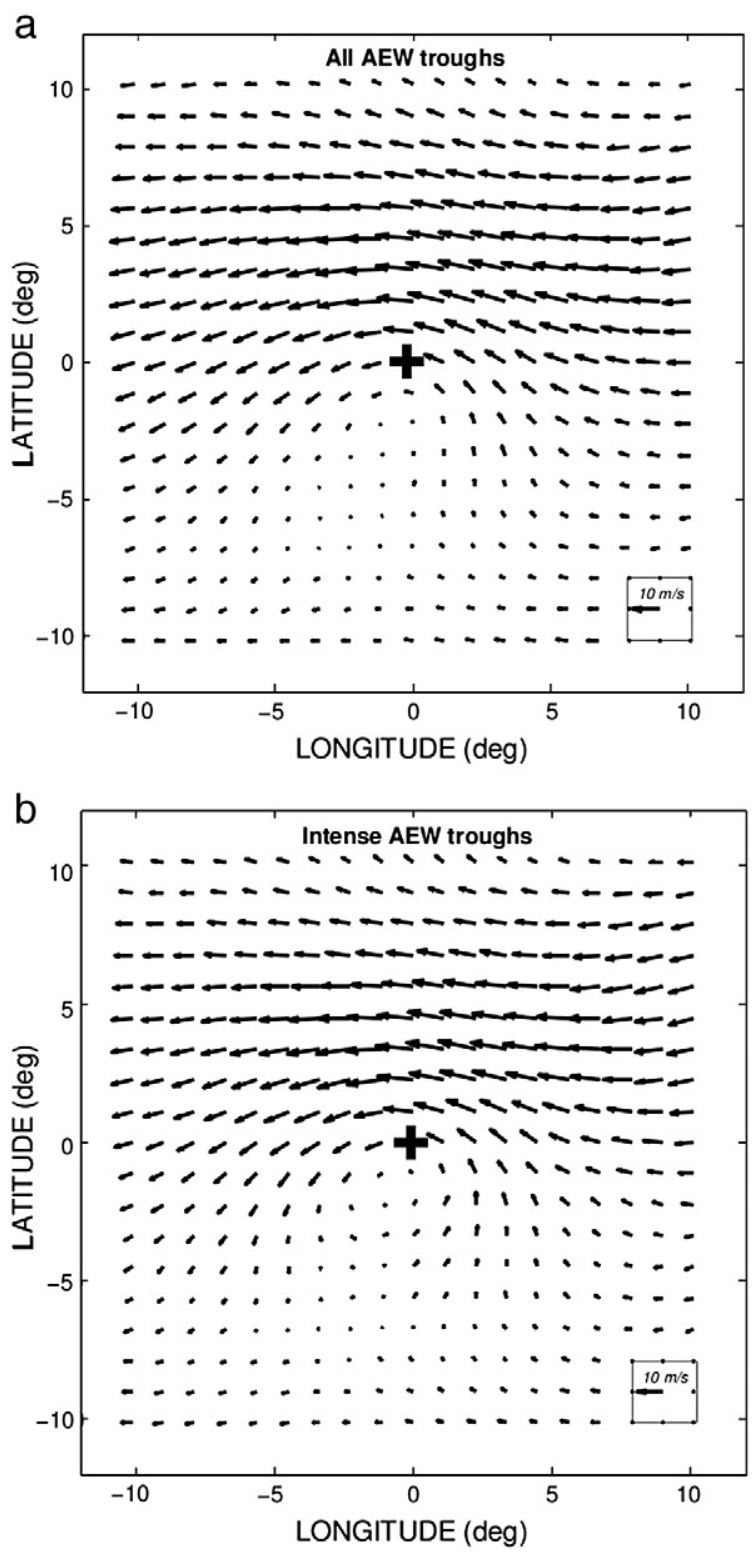

Fig. 3. (a) mean horizontal winds at $700 \mathrm{hPa}$, relative to the respective vorticity centers, for the 142 AEW troughs detected in JAS 04-08. The scale for horizontal winds is indicated on the bottom right. (b) as in (a), except for the 54 "intense AEW troughs" detected in JAS 04-08. The "+" symbol indicates the location of the vorticity center at $700 \mathrm{hPa}$.

system of clouds and thunderstorms with a defined, closed surface circulation and maximum 10-min surface winds of less than $17 \mathrm{~m} \mathrm{~s}^{-1}$ ), then into named storms. 105 (74\%) of the $142 \mathrm{AEW}$ troughs detected during the five seasons did not develop. Nine (6\%) developed east of $30^{\circ} \mathrm{W}$, five $(4 \%)$ between 30 and $40^{\circ} \mathrm{W}$, fourteen $(10 \%)$ between 40 and $60^{\circ} \mathrm{W}$, and nine (6\%) west of $60^{\circ} \mathrm{W}$ (Tables $1-5$ ). $77 \%$ of the tropical storms and hurricanes in JAS 04-08 were related to AEW troughs (2004: 10 out of 13; 2005: 12 out of 14 ; 2006: 7 out of 8; 2007: 7 out of 11; and 2008: 8 out of 11). All named tropical disturbances which formed east of $60^{\circ} \mathrm{W}$ were associated with AEW troughs, except Tropical Storms Jerry $\left(36.2^{\circ} \mathrm{N}, 46.1^{\circ} \mathrm{W}\right.$ on 23 September 2007) and Laura $\left(39.0^{\circ} \mathrm{N}, 35.0^{\circ} \mathrm{N}\right.$ on 26 September 2008$)$, but these two storms were rather of subtropical nature. West of $60^{\circ} \mathrm{W}$, several storms had a different origin (local convective development, low-level disturbance associated with a monsoon trough, and tropical transition of mid-latitude systems). The AEW troughs that developed west of $60^{\circ} \mathrm{W}$ evolved over the Atlantic under the influence of local atmospheric and oceanic conditions, not explicitly related to the AEW characteristics, and they will not be discussed here.

In 2005 , Tropical Storm Gert formed at $19.3^{\circ} \mathrm{N}, 92.9^{\circ} \mathrm{W}$ on 23 July in the southern part of AEW trough $n^{\circ} 4$ that previously spawned Tropical Storm Franklin at $25.0^{\circ} \mathrm{N}$, $27.0^{\circ} \mathrm{W}$ on 21 July (Table 2, Beven et al., 2008). In 2006, Hurricane Ernesto developed at $12.7^{\circ} \mathrm{N}, 61.6^{\circ} \mathrm{W}$ on 24 August from an AEW trough that was undistinguishable in domain $\Omega$ on 20-22 August from $n^{\circ} 16$ which triggered Tropical Storm Debby at $11.6^{\circ} \mathrm{N}, 21.7^{\circ} \mathrm{W}$ on 21 August (Table 3, Franklin and Brown, 2008). Likewise, the AEW trough $n^{\circ} 18$ spawned Tropical Storm Florence at $14.1^{\circ} \mathrm{N}, 39.4^{\circ} \mathrm{W}$ on 3 September 2006, then Hurricane Gordon at $20.2^{\circ} \mathrm{N}, 53.8^{\circ} \mathrm{W}$ on 10 September 2006 (Table 3, Franklin and Brown, 2008). These "secondary" developments will not be considered here.

Among the 54 intense AEW troughs detected during the five seasons, eight developed east of $30^{\circ} \mathrm{W}$, five between 30 and $40^{\circ} \mathrm{W}$, four between 40 and $60^{\circ} \mathrm{W}$, and five west of $60^{\circ} \mathrm{W}$ (Table 2). East of $40^{\circ} \mathrm{W}$, only one named storm (Irene in 2005) out of 15 was not related to an intense AEW trough ( $\mathrm{n}^{\circ} 14$ of 2005), whereas 18 out of the 27 storms west of $40^{\circ} \mathrm{W}$ were triggered by "non intense" AEW troughs. This shows that the intensity of AEW troughs is important for cyclogenesis in the Cape Verde Islands region and the eastern tropical Atlantic, but not further west.

Also seen in Tables $1-5$, the intense AEW troughs that did not develop are relatively more frequent during the first half of the considered period (1 July-14 August). This could result from an active West African monsoon generating strong AEWs which however did not develop over the Atlantic Ocean due to unfavourable environmental conditions in the early season. It is also remarkable that the very active hurricane season of 2005 (Table 2) had the largest number (32) of AEWs, but no tropical cyclogenesis in the Cape Verde Islands region $\left(<30^{\circ} \mathrm{W}\right)$, and that only four intense AEW troughs were observed, none of which having evolved into a named storm east of $60^{\circ} \mathrm{W}$. During this very unusual year, only Tropical Storm Irene developed east of $40^{\circ} \mathrm{W}$ from an AEW trough of moderate intensity.

The fact that only 13 (24\%) of 54 intense AEW troughs developed east of $40^{\circ} \mathrm{W}$ and that some of the strongest AEW troughs (AEW n ${ }^{\circ} 20$ in $2005, n^{\circ} 12$ in 2006, $n^{\circ} 9$ in 2007, $n^{\circ} 7$ and $\mathrm{n}^{\circ} 10$ in 2008) did not evolve into named storms indicates that, as proposed by Carlson (1969a), there is no strict correlation between the intensity of an AEW trough leaving the West African coast and cyclogenetic evolution over the tropical Atlantic Ocean. Then, for the data considered here, the presence of such an intense AEW trough can be considered as a necessary, but not sufficient, condition for cyclogenetic development in the Cape Verde Islands region. 


\section{Low-level vorticity off the West African coast}

4.1. Interaction between monsoon flow and trade winds in the low levels

The low-level circulation off the West African coast, with the south-westerly monsoon flow and the north-easterly trade winds, plays an important role in cyclogenesis. The lowlevel flow can favour (or not) convective developments in the presence of a mid-level vortex (e.g. Fritsch et al., 1994), or facilitate (or not) the up-scale organization of convective cyclonic vortices (e.g. Van Sang et al., 2008).

Cape Verde cyclones can develop from relatively dry and shallow "northern vortices", or from the merging of vortices from northern and southern AEW tracks (e.g. Berry and Thorncroft, 2005; Pytharoulis and Thorncroft, 1998; Ross and
Krishnamurti, 2007; Schwendike and Jones, 2010). Though not frequent, such a scenario can be illustrated with the case of AEW trough $\mathrm{n}^{\circ} 14$ in 2004 which became a tropical depression at $12.3^{\circ} \mathrm{N}, 21.8^{\circ} \mathrm{W}$ on 13 August, and Hurricane Danielle later on (Fig. 4). The south-westerly monsoon flow and the north-easterly oceanic trade winds were rather weak and disconnected from each other on 9 August, three days prior to cyclogenesis (Fig. 4a). On 11 August in the afternoon, a northern vortex originating from the Saharan heat low was centered near $15^{\circ} \mathrm{N}, 15^{\circ} \mathrm{W}$ and it was associated with a relative minimum in surface pressure. This vortex moved south-westward in association with the oceanic trade winds, while the monsoon flow was becoming stronger (Fig. 4c and d). This resulted in a large low-level cyclonic circulation, then the formation of a tropical depression on 13 August, and Tropical Storm Danielle on 14 August (Fig. 4e).
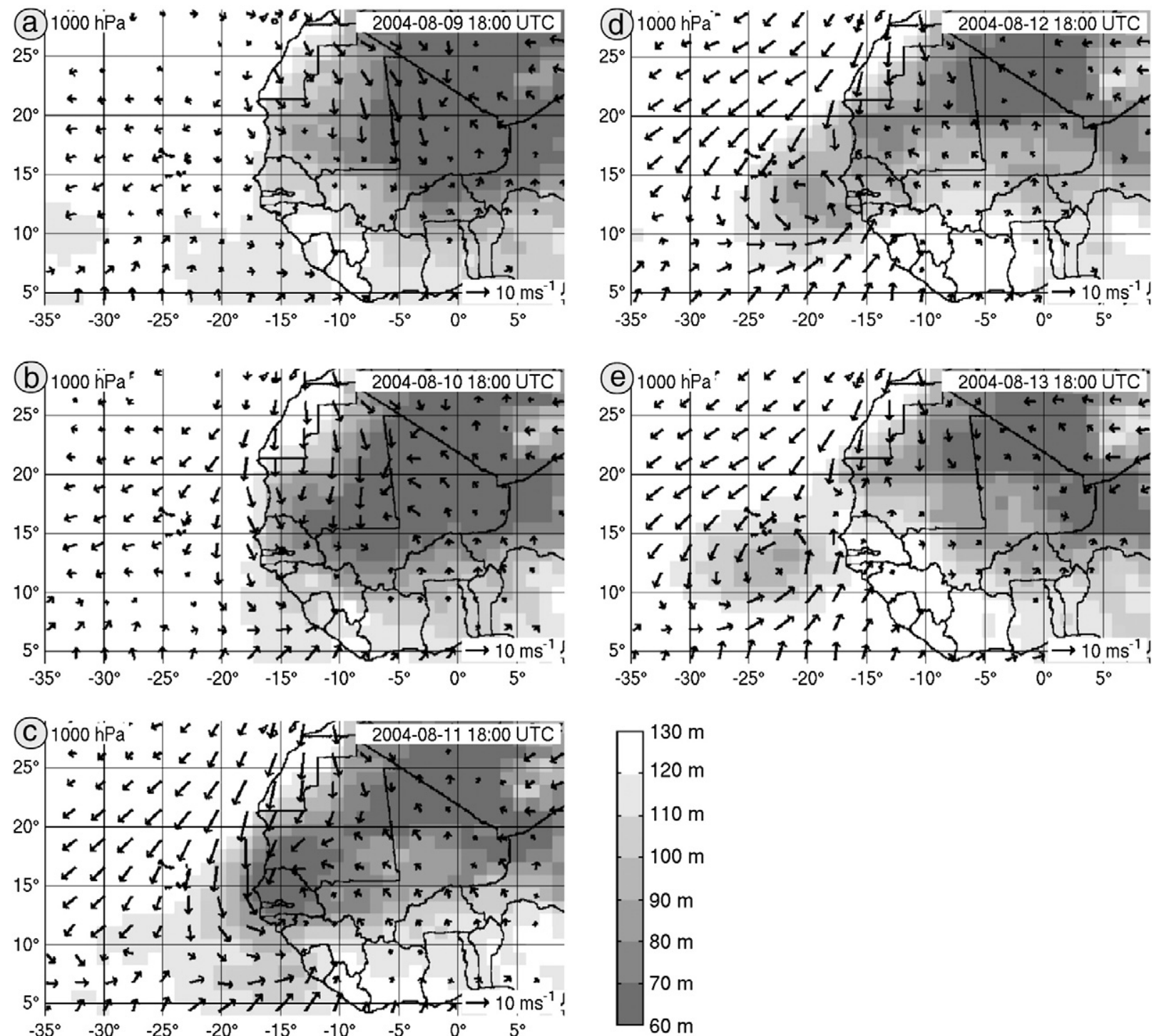

Fig. 4. Horizontal winds (arrows) and geopotential height (m) at $1000 \mathrm{hPa}$ associated with AEW n 14 of 2004 from (a) 18 UTC 9 August until (e) 18 UTC 13 August with a time interval of $24 \mathrm{~h}$. The scale for geopotential height of $1000 \mathrm{hPa}$ is indicated in the right of panel (c). The scale for horizontal winds is indicated in the bottom right of each panel. The trough centered near $13^{\circ} \mathrm{N}, 22^{\circ} \mathrm{W}$ on 18 UTC 13 August (e) is the tropical depression that became Hurricane Danielle (2004). 
Interaction between trade winds and monsoon flow does not depend on northern vortices only. As an example, AEW trough $n^{\circ} 24$ of 2004 became a tropical depression near $11^{\circ} \mathrm{N}$, $29^{\circ} \mathrm{W}$ on 16 September, then Hurricane Karl (Fig. 5). A few days prior to cyclogenesis (Fig. $5 \mathrm{a}$ and b), a weak northern low-level vortex was located near $20^{\circ} \mathrm{N}, 15^{\circ} \mathrm{W}$. The oceanic trade winds were well established, but the monsoon flow was very weak. On 14 September in the afternoon (Fig. 5c), a lowlevel vortex had formed further south, near $10^{\circ} \mathrm{N}, 20^{\circ} \mathrm{W}$, in relation with convective development over warm waters (not shown). This vortex was associated with a stronger monsoon flow. As it propagated westward on 15 September, this vortex went close to the trade winds to its north-west, which resulted in a large cyclonic circulation and the formation of Tropical Storm Karl (Fig. 5d and e).

These two examples show that north-easterly trade winds and south-westerly monsoon flow play an important role in tropical cyclogenesis off West Africa as, at the interface between these two oppositely directed flows, a small-scale vortex can evolve into a large cyclonic circulation which provides favourable low-level environment for tropical cyclogenesis.

\subsection{AEW troughs with low-level cyclonic circulation}

We identify AEW troughs associated with a low-level cyclonic circulation through the simultaneous presence of relative vorticity values at $1000 \mathrm{hPa}$ larger than $10^{-4} \mathrm{~s}^{-1}$ in domain $\Omega$ (this threshold is close to the uppermost percentile of the vorticity values at $1000 \mathrm{hPa}$ for JAS 04-08), and relative vorticity values larger than $+5 \times 10^{-5} \mathrm{~s}^{-1}$ at $700 \mathrm{hPa}$. This last constraint ensures that the low-level cyclonic vorticity is collocated with an AEW trough detected at $700 \mathrm{hPa}$. The verification of this criterion during more than $24 \mathrm{~h}$ defines a
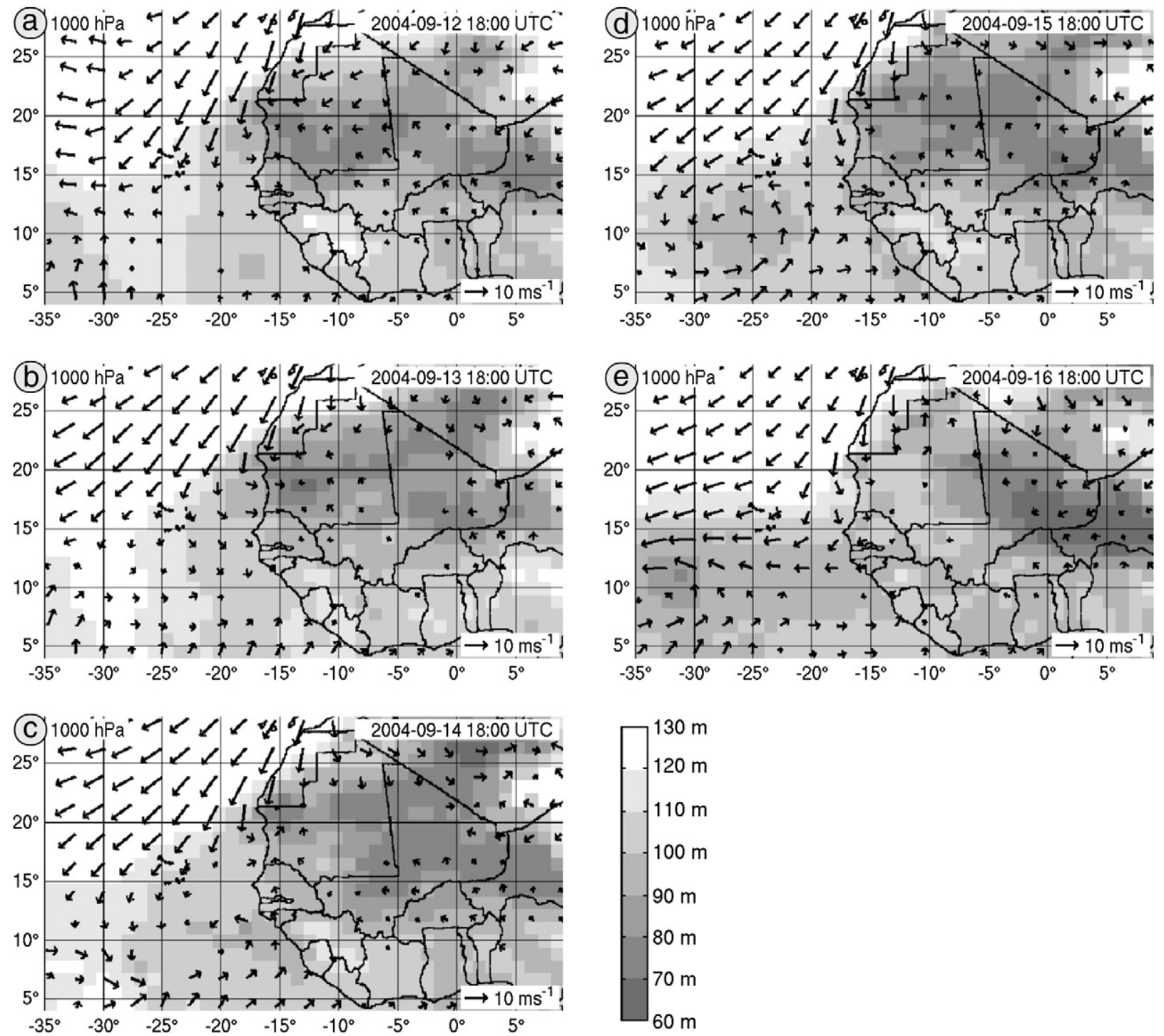

Fig. 5. As in Fig. 4, except for AEW trough $n^{\circ} 24$ of 2004 from (a) 18 UTC 12 September until (e) 18 UTC 16 September. The trough centered near $11^{\circ} \mathrm{N}, 31^{\circ} \mathrm{W}$ on 18 UTC 16 September (e) is the tropical depression that became Karl (2004). 
period of "low-level cyclonic circulation associated with an AEW trough". The examples above show that there is no general rule for the origin of low-level cyclonic vortices. Northern vortices of Saharan origin or southern ones probably related to long-lived MCSs can play this role. Tables 1-5 also reveal that there is no strict correlation between the intensity of an AEW trough at $700 \mathrm{hPa}$ and the presence of cyclonic circulation at $1000 \mathrm{hPa}$.

In JAS 2004, 2005, 2006, 2007 and 2008, 7, 0, 4, 4 and 6 AEW troughs were associated with low-level cyclonic circulation, respectively (Tables $1-5$ ). The specific nature of 2005 appears again with the lack of low-level circulation associated with AEW troughs (Table 2). The averaged circulation at $1000 \mathrm{hPa}$ (relative to the respective vorticity centers at $700 \mathrm{hPa}$ ) for the 142 AEW troughs of JAS 04-08 is compared in Fig. 6 with that of the 21 AEW troughs associated with low-level cyclonic circulation. For the mean AEW trough (Fig. 6a), the oppositely directed north-easterly trade winds and south-westerly monsoon flow in domain $\Omega$ are separated by a 100 - to $200-\mathrm{km}$ wide zone of calm winds and relatively low vorticity. For the $21 \mathrm{AEW}$ troughs with low-level cyclonic circulation, there is a large zone of swirling motions at the interface between the two flows (Fig. 6b). Moreover, as seen when comparing Figs. $3 \mathrm{~b}$ and $6 \mathrm{~b}$, the low and mid-level vorticity centers are vertically aligned, a favourable condition for tropical cyclogenesis.

Among the 21 AEW troughs associated with low-level cyclonic circulation in JAS 04-08, nine (43\%) developed east of $30^{\circ} \mathrm{W}$, four (19\%) between 30 and $40^{\circ} \mathrm{W}$, one (5\%) between 40 and $60^{\circ} \mathrm{W}$, and two (10\%) west of $60^{\circ} \mathrm{W}$ (Tables 1-5). Only two storms (Irene in 2005, and Karen in 2007) out of fourteen which formed west $40^{\circ} \mathrm{W}$ in JAS 04-08 were not associated with an AEW trough with low-level cyclonic circulation off the West African coast. Five (23\%) AEW troughs with lowlevel circulation ( $n^{\circ} 6$ and 20 in 2004, 17 in 2007, 10 and 13 in 2008) did not develop. This suggests that having the SW monsoon flow and the NE maritime trade winds close to each other off the west coast of Africa is an important (but not necessary) condition for cyclogenetic evolution in the eastern part of the tropical Atlantic Ocean (between 30 and $40^{\circ} \mathrm{W}$ ), and a necessary (but not sufficient) one in the Cape Verde Islands region (east of $30^{\circ} \mathrm{W}$ ).

It is remarkable that all the AEW troughs with strong lowlevel cyclonic vorticity at $1000 \mathrm{hPa}$, except $\mathrm{n}^{\circ} 11$ in 2007 which evolved in Tropical Storm Erin west of $60^{\circ} \mathrm{W}$, also had intense cyclonic vorticity at $700 \mathrm{hPa}$. They can therefore be identified as "intense AEW troughs with low-level cyclonic circulation". The reasons why seven (35\%) of these 20 AEW troughs did not develop east of $40^{\circ} \mathrm{W}$ are to be found either in the influence of a nearby anticyclonic circulation, the lack of intense convective activity, or adverse environmental conditions over the ocean.

\section{Interaction with the Saharan anticyclone}

The influence of Saharan air advected over the tropical Atlantic Ocean on cyclogenesis is still an open question (e.g. Braun, 2010). The presence of dry and dusty air at mid-levels can either increase the strength of the AEJ and favour AEW growth through barotropic and/or baroclinic instability, modify cloud microphysics and convective development, or
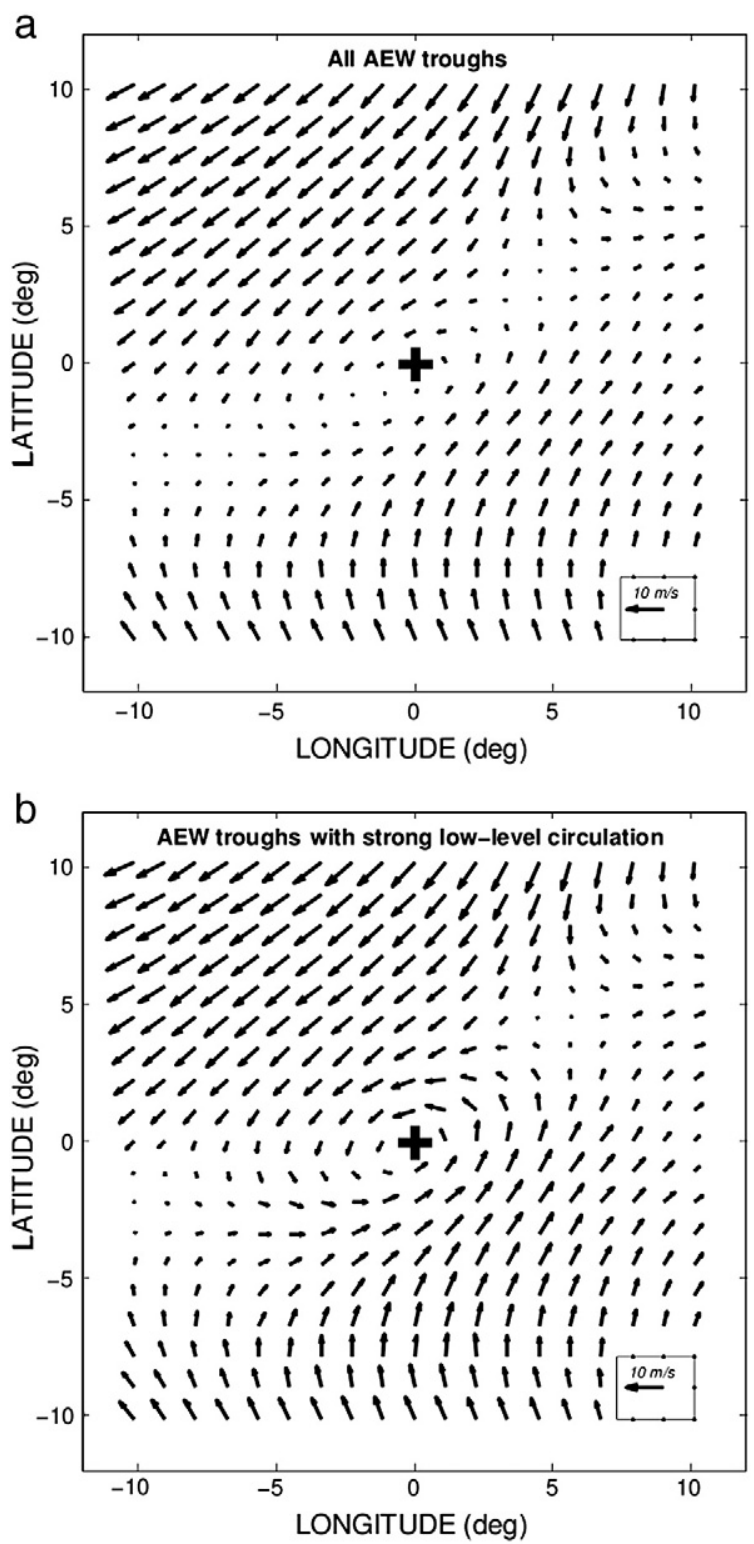

Fig. 6. (a) mean horizontal winds at $1000 \mathrm{hPa}$, relative to the respective vorticity centers at $700 \mathrm{hPa}$, for the 142 AEW troughs detected in JAS 04-08. (b) as in (a), except for the 21 "AEW troughs associated with strong lowlevel cyclonic vorticity". The scale for horizontal winds is indicated in the bottom right of each panel. The "+" symbol indicates the location of the vorticity center at $700 \mathrm{hPa}$.

inhibit cyclogenetic evolution owing to enhanced low-level temperature inversion, increased vertical wind shear, and/or drier troposphere. We will focus here on the dynamic influence of anticyclonic circulation atop the Saharan boundary layer which occasionally reinforces AEW ridges and interact with cyclogenetic evolution in downstream (western) AEW troughs.

\subsection{Vortex disorganization by mid-level anticyclonic Saharan air}

Arnault and Roux (2010) showed that the failed cyclogenetic evolution of intense AEW trough $\mathrm{n}^{\circ} 25$ on 27-29 
September 2006 was due to an interaction with a strong upstream (eastern) AEW ridge with dry Saharan anticyclonic flow. We will verify whether such unfavourable synoptic conditions can, at least partially, explain the non-developing cases otherwise associated with previously described favourable conditions at low and mid levels.

As an illustration, intense AEW trough $n^{\circ} 10$ of 2008 was associated with strong low-level cyclonic circulation, but failed to develop (Fig. 7). A well defined cyclonic circulation centered on $15^{\circ} \mathrm{N}, 23^{\circ} \mathrm{W}$ is observed at $700 \mathrm{hPa}$ on 30 July 2008 (Fig. 7a, b). At that time, the anticyclonic flow originating from the Saharan region induced strong southeasterly winds northeast of the AEW trough at mid-levels. This anticyclonically rotating south-easterly flow intensified on $31 \mathrm{July}$, stretched the cyclonic vorticity structure of the AEW trough northward (Fig. 7d, e, f) and significantly reduced its intensity (compare Fig. 7a and f). In this case, the Saharan anticyclonic circulation was apparently related to a zone of very dry air over Lybia (not shown). Roca et al. (2005) showed that such dry air structures frequently originated in the upper levels on the anticyclonic side of the polar jet stream at $50^{\circ} \mathrm{N}$. Strong AEW ridges can also result from the spatial and temporal variability of the Saharan heat low (Lavaysse et al., 2010).

\subsection{AEW troughs associated with mid-level anticyclonic flow}

The presence of mid-level anticyclonic circulation associated with the upstream ridge of an AEW in the Cape Verde Island region can be identified with the relative area of southerly winds at $700 \mathrm{hPa}$ stronger than $10 \mathrm{~m} \mathrm{~s}^{-1}$ in the northern part (20-22 $2^{\circ}, 20-28^{\circ} \mathrm{W}$; see Fig. 7 ) of domain $\Omega$. This threshold value is close to the uppermost decile of meridional wind values in this sub-domain for JAS 04-08. The fulfilment of this criterion during more than $24 \mathrm{~h}$ defines a period of "mid-level anticyclonic circulation".

In JAS of 2004, 2005, 2006, 2007 and 2008, 7, 4, 7, 4, and 10 AEW troughs were associated with mid-level anticyclonic circulation, respectively. As seen in Tables 1-5, strong upstream anticyclonic circulations can coexist with strong AEW troughs at mid and, sometimes, low levels: mid-level anticyclonic circulations were associated with intense AEW troughs $\mathrm{n}^{\circ} 27$ in 2004, $\mathrm{n}^{\circ} 20$ in 2005, $\mathrm{n}^{\circ} 7$ and 25 in 2006, and $n^{\circ} 26$ in 2008, as well as intense AEWs troughs with low level cyclonic circulation $n^{\circ} 10,14$ and 19 in 2008. Examination of Tables 1-5 also reveals that anticyclonic circulations of Saharan origin occurred more frequently during the second half of the season (15 August-30 September). This can be related to a slightly weaker Saharan heat low and stronger influence of the northern subsiding branch of the Hadley circulation, as the equinox is approaching. Also, the progressive reinforcement of the mid-latitudes circulation at the end of boreal summer could generate favourable conditions for extra-tropical dry intrusions through North Africa.

The averaged horizontal wind field at $700 \mathrm{hPa}$ (relative to the respective vorticity centers) for the 142 AEW troughs of JAS 04-08 (Fig. 8a) shows an almost zonal flow north of the vorticity center. For the 32 AEW troughs with mid-level anticyclonic flow (Fig. 8b), the stronger southeasterly flow results from a more intense upstream (eastern) AEW ridge, in relation with a well defined mid-level Saharan anticyclone. It is remarkable that the averaged low-level circulation (relative to the respective vorticity centers at $700 \mathrm{hPa}$ ) for those AEWs with a mid-level anticyclonic flow (Fig. 8c) is shifted $200-300 \mathrm{~km}$ south of the mid-level vorticity center (Fig. 8b). Only one of the 32 AEW troughs with mid-level anticyclonic circulation developed: intense AEW trough $\mathrm{n}^{\circ} 18$ in 2008 with low level circulation became Hurricane Ike at $17.2^{\circ} \mathrm{N}, 37.0^{\circ} \mathrm{W}$.

\subsection{Intense AEW troughs with low-level cyclonic circulation} and weak mid-level anticyclonic flow

Among the eight intense AEW troughs with low-level cyclonic circulation that did not develop east of $40^{\circ} \mathrm{W}$ in JAS 04-08, two ( $\mathrm{n}^{\circ} 10$ and 14 in 2008) were associated with Saharan anticyclonic flow at $700 \mathrm{hPa}$ (Table 5). All tropical depressions, then named storms, which developed in the Cape Verde Islands region (east of $30^{\circ} \mathrm{W}$ ) were characterized by the simultaneous presence of an intense AEW trough and cyclonic circulation in the low levels, and absence of anticyclonic Saharan flow in the mid-troposphere for more than $24 \mathrm{~h}$. These necessary (but still not sufficient) conditions for tropical cyclogenesis are referred to as "favourable synoptic conditions".

Among the 17 AEW troughs associated with these "favourable synoptic conditions" in JAS 04-08, nine developed east of $30^{\circ} \mathrm{W}$, three between 30 and $40^{\circ} \mathrm{W}$, and one between 40 and $60^{\circ} \mathrm{W}$ (Tables $1-5$ ). The fact that four (24\%) AEW troughs satisfying the above criteria did not develop shows that the presence of favourable synoptic conditions is not sufficient for tropical cyclogenesis to occur in the Cape Verde Islands region and over the tropical eastern Atlantic.

\section{Convective processes}

\subsection{Cape Verde tropical cyclogenesis and convective processes}

Convective processes are involved in the development and intensification of AEWs over West Africa and the eastern tropical Atlantic. Low-level cyclonic vorticity between the south-westerly monsoon flow and the north-easterly trade winds, frequently associated with Cape Verde tropical cyclogenesis, can be triggered by convective processes. MCVs forming in the mid levels of stratiform region of MCSs can be precursors of tropical cyclogenesis (e.g. Bister and Emanuel, 1997). Also, as suggested by, e.g., Van Sang et al. (2008), tropical cyclogenesis could result from up-scale organization of stochastically produced convective-scale cyclonic vortices embedded in a large-scale cyclonically rotating flow. Arnault and Roux (2009) analyzed the associated intensification of a low to mid level cyclonic vortex in the Cape Verde Islands area, then the formation of Helene (2006), as the consequence of a geostrophic adjustment following convectively induced pressure decrease. Likewise, Ross et al. (2009) found that developing waves possess positive barotropic energy conversion in conjunction with diabatic heating related to enhanced convective activity. In order to investigate the contribution of convective processes 

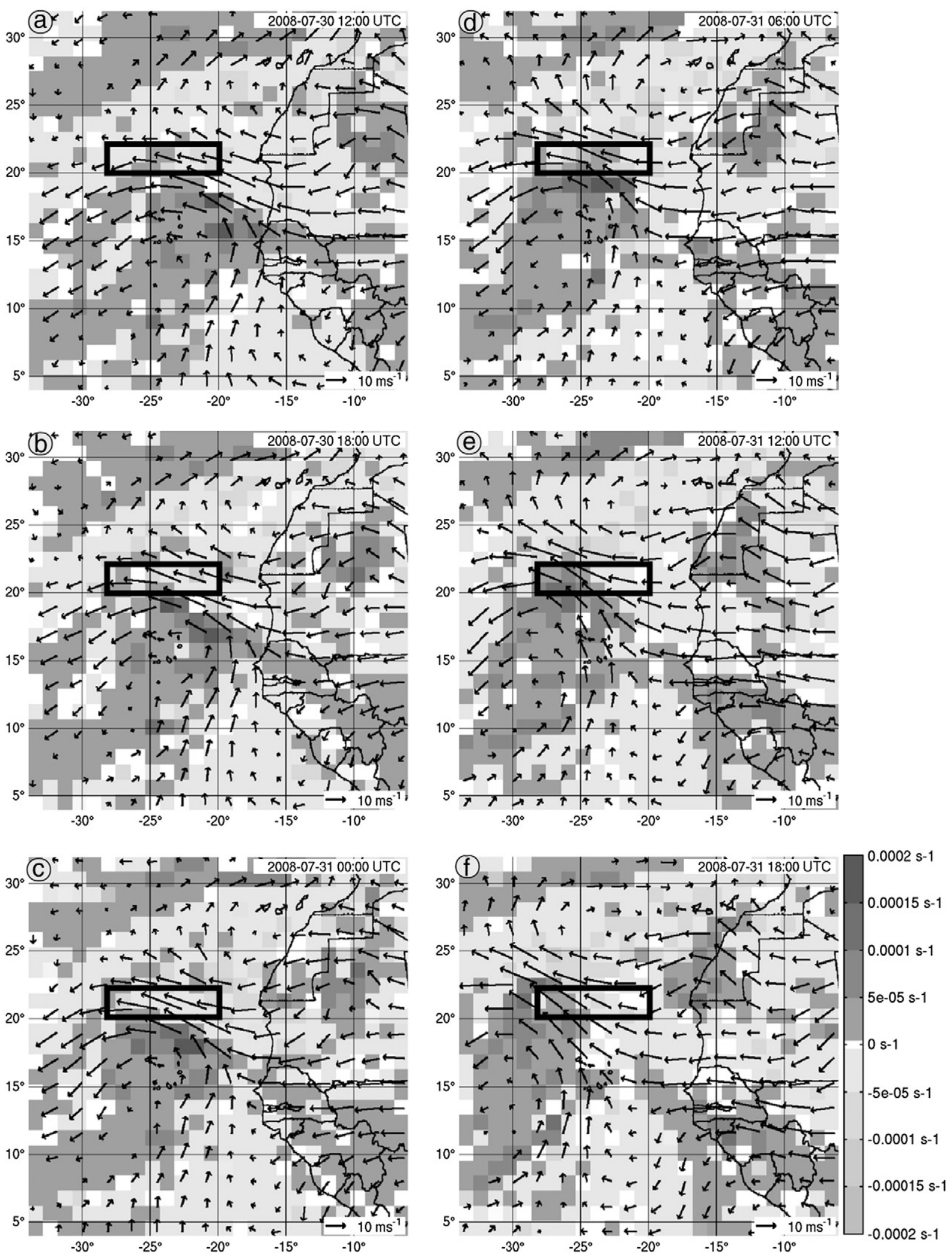

Fig. 7. Horizontal winds (arrows) and relative vorticity $\left(\mathrm{s}^{-1}\right)$ at $700 \mathrm{hPa}$ associated with AEW $\mathrm{n}^{\circ} 10$ of 2008 , from (a) 12 UTC 30 July until (f) 18 UTC 31 July with a time interval of $6 \mathrm{~h}$. The black rectangle indicates the northern part of domain $\Omega$ used for the criterion to detect strong mid-level anticyclonic flow. Vorticity scale is indicated in the bottom right. The scale for horizontal wind is indicated in the bottom right of each panel. 
in Cape Verde tropical cyclogenesis, we analyze the convective activity associated with the 142 AEW troughs of JAS 04-08.
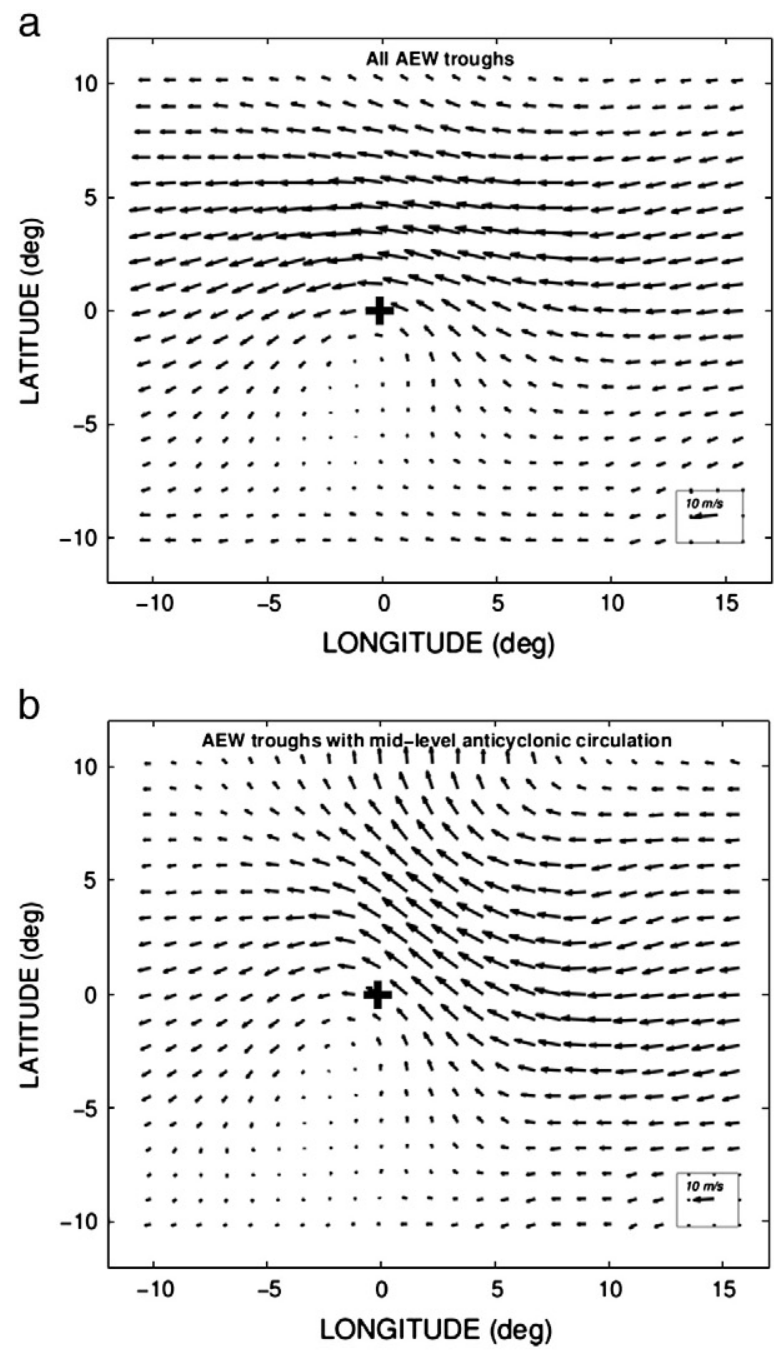

C

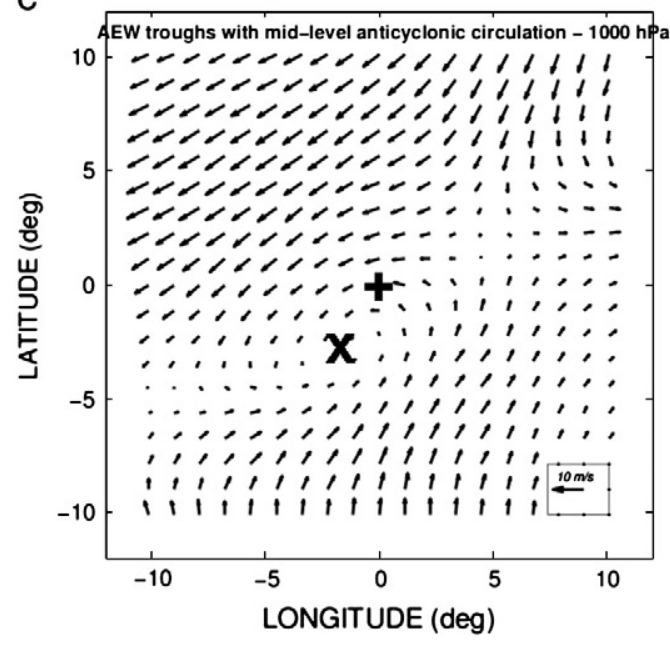

\subsection{AEW troughs with deep convective activity}

The hourly cloud top brightness temperatures measured by Meteosat- 9 in the water vapour channel $n^{\circ} 6$ are averaged over 6-h periods centered on ECMWF analysis times to facilitate comparison between the two data sets. Here, we consider that AEW troughs are associated with substantial convective activity when cloud top brightness temperatures $\left(\mathrm{T}_{\mathrm{B}}\right)$ colder than $-50{ }^{\circ} \mathrm{C}$ are found in domain $\Omega$. $\mathrm{T}_{\mathrm{B}}$ values colder than $-50{ }^{\circ} \mathrm{C}$ represent less than $1 \%$ (lower percentile) of all values for JAS 04-08. The verification of this criterion for more than $24 \mathrm{~h}$ defines a period of "deep convective activity associated with an AEW trough".

Fig. 9 shows that not all the AEW troughs were associated with intense convective activity. In JAS of 2004, 2005, 2006, 2007 and 2008, 9, 7, 8, 10 and 8 AEW troughs verified this criterion, respectively. The average brightness temperatures associated with the 142 AEW troughs of JAS 04-08, relative to their respective vorticity centers, (Fig. 9a) are compared with those for the $39 \mathrm{AEW}$ troughs with deep convective activity (Fig. 9b). It must be noted that the $T_{B}$ values in Fig. 9 are slightly warmer than those of individual cases as they are averaged for several AEW troughs with slightly different convective organizations. The mean $T_{B}$ values associated with the 142 AEW troughs (Fig. 9a) reveal the presence of the west-southwest-east-northeast oriented inter-tropical convergence zone (ITCZ) south of the vorticity center, with minimum $\mathrm{T}_{\mathrm{B}}$ values $<-28^{\circ} \mathrm{C}$. The cloud top signature of the 39 AEW troughs with deep convective activity (Fig. 9b) is more pronounced, with colder $T_{B}$ values $<-36{ }^{\circ} \mathrm{C}$ southeast of the vorticity center. This shows that enhanced convection occurred in connection with the AEW troughs and did not result from randomly distributed activity in the ITCZ. Fig. 9c shows the average brightness temperatures associated with the $32 \mathrm{AEW}$ troughs with mid-level anticyclonic circulation. Convective developments are inhibited east and north of the vorticity center owing the presence of dry air in the mid levels and occur hundreds of kilometers to the south and southwest, a situation obviously unfavourable to cyclogenesis.

\subsection{AEW troughs associated with favourable synoptic conditions and deep convective activity}

Only three ( $\mathrm{n}^{\circ} 6$ and 17 in 2004, and $n^{\circ} 23$ in 2006) of the $17 \mathrm{AEW}$ troughs with "favourable synoptic conditions" were not associated with deep convective activity, suggesting that such an environment either favours sustained convective activity or could result from it. However, the fact that two thirds (28 among 42) of the AEW troughs with deep convection were not associated with "favourable synoptic conditions", and only two of these developed into named storms east of $60^{\circ} \mathrm{W}\left(\mathrm{n}^{\circ} 14-\right.$ Irene in 2005 at $12.9^{\circ} \mathrm{N}, 33.5^{\circ} \mathrm{W}$; and $\mathrm{n}^{\circ} 29$ - Karen in 2007 at $10.0^{\circ} \mathrm{N}, 35.9^{\circ} \mathrm{W}$ ), indicates that

Fig. 8. (a) mean horizontal winds (arrows) at $700 \mathrm{hPa}$, relative to the respective vorticity centers, for the 142 AEW troughs detected in JAS 04-08. (b) as in (a), except for the 32 "AEW troughs associated with strong midlevel anticyclonic flow". (c) as in (b), except for $1000 \mathrm{hPa}$ level. The scale for horizontal winds is indicated in the bottom right corner of each panel. The "+" and " $\mathrm{x"}$ "symbols indicate the location of the vorticity center at 700 and $1000 \mathrm{hPa}$, respectively. 
convection in an AEW trough is not a sufficient condition and that dynamic processes play a crucial role in Cape Verde tropical cyclogenesis. This confirms the idea by Sall et al. (2006) that convective systems observed off the West African

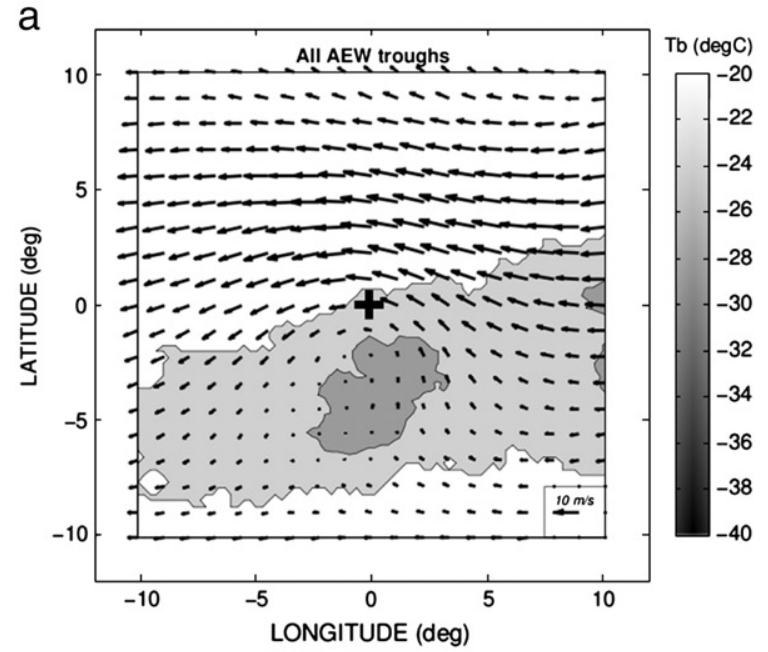

b

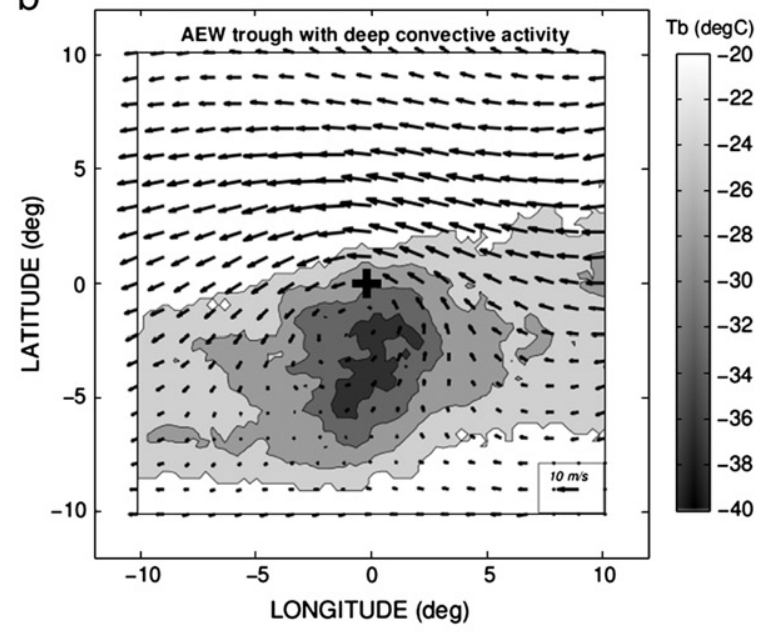

C

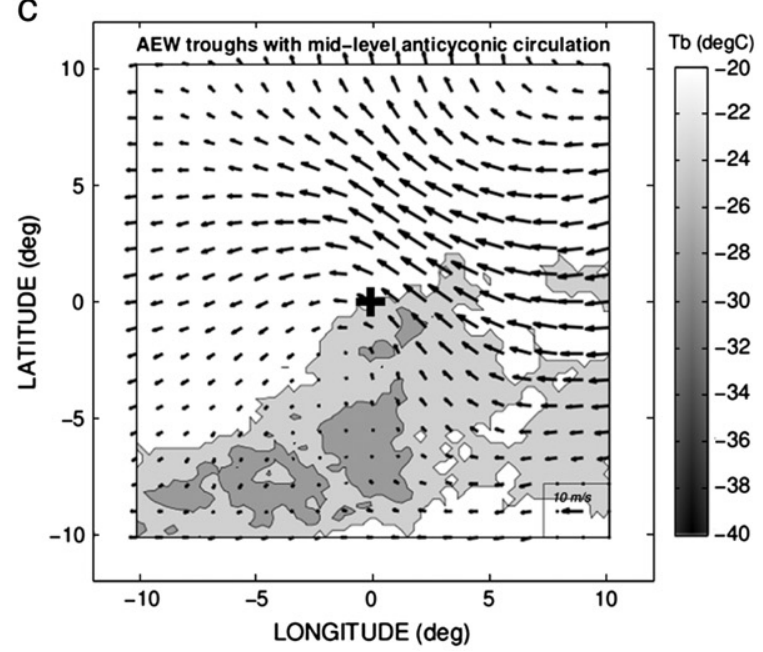

coast have a cyclogenetic evolution only when they encounter a favourable synoptic environment in this region.

Among the 14 AEW troughs associated with "favourable synoptic and convective conditions" during the five seasons, 9 developed east of $30^{\circ} \mathrm{W}$ and spawned all the storms that formed there in JAS 04-08 ( $n^{\circ} 14$ - Danielle, $n^{\circ} 19$ - Ivan and $\mathrm{n}^{\circ} 24 \mathrm{Karl}$ in 2004; $\mathrm{n}^{\circ} 16$ - Debby and $\mathrm{n}^{\circ} 20$ - Helene in 2006; $n^{\circ} 14-$ Dean and $n^{\circ} 27$ - Melissa in 2007; $n^{\circ} 1$ - Bertha and $\mathrm{n}^{\circ} 19$ - Josephine in 2008), two ( $\mathrm{n}^{\circ} 25$ - Lisa in 2004; and $\mathrm{n}^{\circ} 18$ - Florence in 2006) evolved between 30 and $40^{\circ} \mathrm{W}$, three ( $n^{\circ} 20$ in $2004, n^{\circ} 17$ in 2007, and $n^{\circ} 13$ in 2008) did not develop (Tables 1-5). Therefore, for the data set considered here, the simultaneous presence of favourable synoptic and convective conditions is a necessary, but still not an absolutely sufficient, factor for Cape Verde tropical cyclogenesis (east of $30^{\circ} \mathrm{W}$ ).

\section{Sea surface temperature, tropospheric humidity and vertical wind shear}

Warm ocean waters, relatively moist mid-troposphere and weak tropospheric wind shear are necessary environmental factors for tropical cyclogenesis. Sall et al. (2006) and Wang et al. (2009) brought evidence that SST and vertical wind shear are not the most important discriminating factors for Cape Verde cyclogenesis. Nevertheless, we will seek whether environmental characteristics could reveal why the three AEW troughs with "favourable synoptic and convective conditions" ( $n^{\circ} 20$ in 2004, $n^{\circ} 17$ in 2007, and $n^{\circ} 13$ in 2008) did not develop.

\subsection{Cape Verde tropical cyclogenesis and sea surface temperature}

Warm SST and oceanic heat content are necessary conditions for tropical cyclogenesis as sensible and, more important, latent heat fluxes from the ocean surface provide unstable air feeding convective upward motions which produce precipitation and release latent heat in the troposphere. Fig. 10a shows the distribution of the mean SST values in domain $\Omega$ for the 142 AEW troughs of JAS 04-08. All values are warmer than $25^{\circ} \mathrm{C}$, and about two thirds are above $26^{\circ} \mathrm{C}$. The three non-developing AEWs had SSTs warmer than $26^{\circ} \mathrm{C}$ (up to $27.3^{\circ} \mathrm{C}$ for $\mathrm{AEW} \mathrm{n}^{\circ} 20$ of 2004). This confirms the result by Sall et al. (2006) that SST does not allow discrimination between developing and non-developing cases in the Cape Verde Islands region.

\subsection{Cape Verde tropical cyclogenesis and tropospheric humidity}

A dry mid-troposphere is an inhibiting factor for tropical cyclogenesis as it permits the development of cold convective downdrafts through evaporative cooling. Fig. 10b shows the

Fig. 9. (a) mean horizontal winds (arrows) at $700 \mathrm{hPa}$ and mean brightness temperature $\left({ }^{\circ} \mathrm{C}\right.$ ) for the 142 AEW troughs detected in JAS 04-08. (b) as in (a), except for the 39 "AEW troughs associated with deep convective activity". (c) as in (a), except for the 32 "AEW troughs with strong mid-level anticyclonic flow". Brightness temperature scale is indicated. The scale for horizontal winds is indicated in the bottom right of each panel. The "+" symbol indicates the location of the vorticity center at $700 \mathrm{hPa}$. 

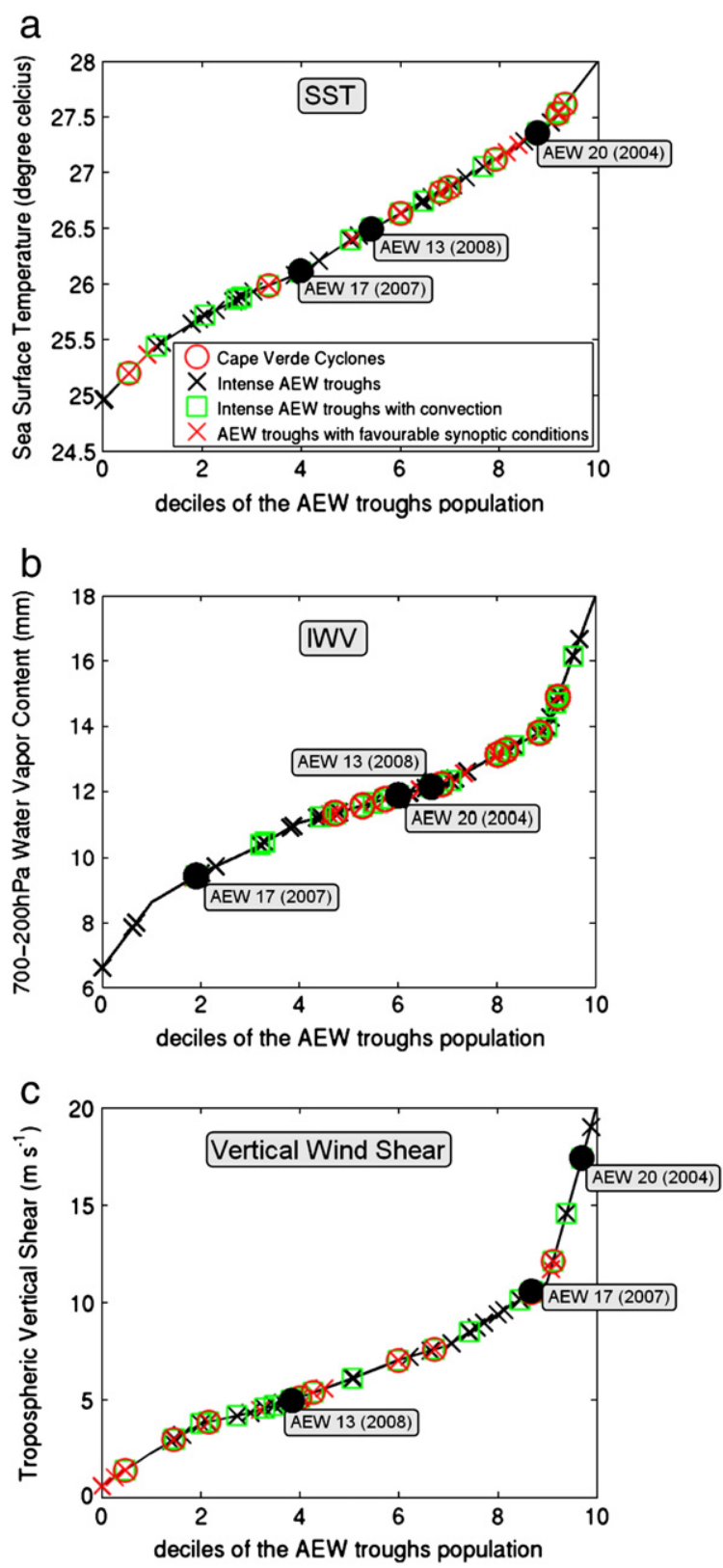

Fig. 10. (a) distribution of averaged sea surface temperatures (SST) values in domain $\Omega$ associated with the 142 AEW troughs of JAS 04-08. SST values associated with Cape Verde storms are indicated with red circles, "intense AEW troughs" with black crosses, "AEW troughs with favourable synoptic environment" with red crosses, "AEW troughs with convective activity" with green squares. The three cases with "favourable synoptic and convective conditions", but did not develop east of $30^{\circ} \mathrm{W}$, are indicated with black dots. (b) as in (a), except for integrated water vapour (IWV) between 700 and $200 \mathrm{hPa}$. (c) as in (a), except for vertical wind shear between 850 and $200 \mathrm{hPa}$.

distribution of the water vapour content integrated between 700 and $200 \mathrm{hPa}$ (IWV), averaged over domain $\Omega$ for the 142 AEW troughs of JAS 04-08. Since moisture in the southwesterly monsoon flow and oceanic boundary layer is large and relatively uniform, the contribution of the lowest $300 \mathrm{hPa}$ is not taken into account. All the waves which led to tropical cyclogenesis are within the moistest half (IWV $>11 \mathrm{~mm}$ ) of the considered population. AEW trough $n^{\circ} 17$ in 2007 was associated with a relatively dry mid-troposphere with an IWV of $9.5 \mathrm{~mm}$, which might explain why it did not develop. Fig. 10b shows that this is however a relatively rare event, as outlined by Wang et al. (2009).

\subsection{Cape Verde tropical cyclogenesis and tropospheric vertical wind shear}

Fig. 10c shows the distribution of the tropospheric vertical wind shear (calculated as the difference between wind vectors at 850 and $200 \mathrm{hPa}$ ) averaged over domain $\Omega$ for the $142 \mathrm{AEW}$ troughs of JAS 04-08. About 90\% (9 lowest deciles) are associated with vertical wind shear values less than $10 \mathrm{~m} \mathrm{~s}^{-1}$, the long-term average value in the Atlantic "main development region" (MDR: $7.5-20^{\circ} \mathrm{N}, 15-85^{\circ} \mathrm{W}$ ) according to Aiyyer and Thorncroft (2006). These authors found a substantial negative effect of vertical wind shear on tropical cyclogenesis in the MDR when the shear was significantly higher than the long-term mean. Hence, the relatively strong shear values associated with AEW troughs $\mathrm{n}^{\circ} 20$ in $2004\left(17 \mathrm{~m} \mathrm{~s}^{-1}\right)$ and 17 in 2007 $\left(10 \mathrm{~m} \mathrm{~s}^{-1}\right)$ could explain why they failed to develop. Nevertheless, Fig. 10c shows that the environment off West Africa is generally characterized by a weak vertical wind shear $\left(<10 \mathrm{~m} \mathrm{~s}^{1}\right)$, as argued by Sall et al. (2006).

There is no evident explanation for the non-development of AEW n ${ }^{\circ} 13$ in 2008. However, Brown et al. (2010) indicated that, in 2008, Hurricane "Gustav formed [as a tropical depression, on 25 August at $00 \mathrm{UTC}$ ] at $13.5^{\circ} \mathrm{N}, 67.4^{\circ} \mathrm{W}$ from a tropical wave [ $\mathrm{n}^{\circ} 14$ in 2008] that moved westward off the coast of Africa on 13 August. After briefly showing signs of organization on 18 August, westerly vertical wind shear prevented significant development during the next several days". It is therefore speculated that, although vertical wind shear was moderate when AEW trough $\mathrm{n}^{\circ} 13$ was in domain $\Omega$ (Fig. 10c), its development could have been inhibited by increasing shear west of $\Omega$. Unknown analyses problems could also have led to overestimate favourable factors and/or underestimate unfavourable factors.

\section{Successful and failed Cape Verde cyclogenesis}

According to previous results and the present work, it appears that a primary condition for tropical cyclogenesis in the Cape Verde Islands area (east of $30^{\circ} \mathrm{W}$ ) is the simultaneous presence of an intense AEW trough at mid level and a low-level cyclonic circulation off the West African coast between the north-easterly trade winds and the southwesterly monsoon flow. Occasionally, a low-level northern vortex of the AEW can trigger such a circulation, although it is not always present. A complementary condition is the presence of deep convection close to the circulation center. Convection removes air mass from the lowest levels, transports it upward and generates an upper level outflow. This leads to a pressure decrease in the low- to mid-levels, and tropical cyclogenesis can eventually result from geostrophic adjustment and barotropic conversion of the mid-tropospheric zonal easterly jet into cyclonic winds (e.g. Arnault and Roux, 2009; Ross et al., 2009). This is schematically depicted in Fig. 11a. 

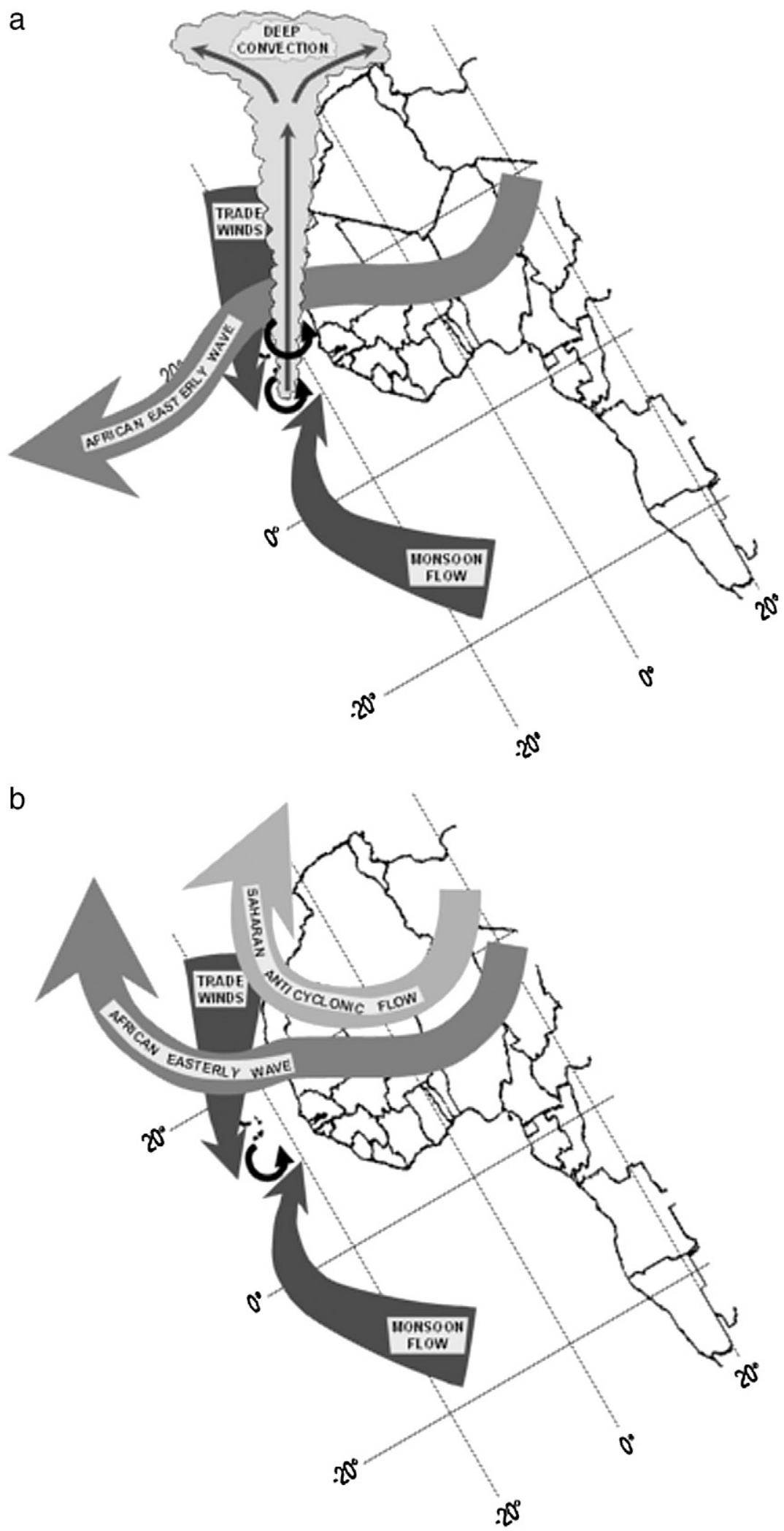

Fig. 11. Schematic view of (a) successful and (b) failed Cape Verde tropical cyclogenesis. 
However, when the anticyclonic flow associated with the upstream AEW ridge to the east is enhanced by mid-level anticyclonic Saharan air, AEW troughs - even when associated with low-level circulation and sustained convection generally fails to develop off the West African coast. Indeed, the strong anticyclonic southeasterly flow elongates the developing cyclonic structure north-westward. This disorganizes the associated mass field, convection and vorticity perturbation, and prohibits cyclogenetic evolution (e.g. Arnault and Roux, 2010). A schematic view of such nondevelopment is displayed in Fig. 11b.

\section{Conclusion and perspectives}

This work aimed at examining the physical processes associated with cyclogenetic evolution of AEW troughs that reached tropical depression intensity in the Cape Verde Islands region (east of $30^{\circ} \mathrm{W}$ ) and eastern tropical Atlantic (30-40 $\left.{ }^{\circ} \mathrm{W}\right)$, then developed into named storms, during JAS 04-08. Among the 142 AEW troughs detected during this period, eleven developing (nine east of $30^{\circ} \mathrm{W}$, two between 30 and $40^{\circ} \mathrm{W}$ ) and three non-developing cases were associated with the simultaneous presence of intense AEW trough and weak anticyclonic Saharan flow at mid level, lowlevel cyclonic circulation between the north-easterly trade winds and the south-westerly monsoon flow, and intense convective activity. No other storm developed east of $30^{\circ} \mathrm{W}$, but four named storms among six were triggered between 30 and $40^{\circ} \mathrm{W}$ by AEW troughs that did not match all these characteristics. Although these results are based on a limited data set, we can speculate that AEW troughs with such characteristics would have a high probability $(11 / 14 \approx 80 \%)$ to develop east of $30^{\circ} \mathrm{W}(9 / 14 \approx 65 \%)$ or between 30 and $40^{\circ} \mathrm{W}(2 / 14 \approx 15 \%)$. Unusually dry environment or strong vertical wind shear can however impede such an evolution with a probability of $\approx 20 \%(3 / 14)$.

More quantitative studies should be conducted to further investigate and improve this conceptual model of Cape Verde tropical cyclone formation. Other processes should also be analyzed, such as: the link between the Saharan heat low and anticyclonic surges at mid-levels (Lavaysse et al., 2010), the influence of Saharan dust on microphysical and dynamic processes (Jenkins et al., 2008), the role played by deep convection in decreasing the pressure in the low to mid-levels and the efficiency of geostrophic adjustment and barotropic/ baroclinic energy conversions, the role of up-scale organization of convective-scale cyclonic vorticity, the influence of airsea fluxes in the successive convective developments, the possible influence of upper-level structures of tropical or mid-latitude origin, etc. ... It would also be useful to extend the present study to a larger data set, such as ECMWF or NCEP/NCAR reanalyses. Comparisons between the most important meso- and large scale conditions leading to tropical cyclogenesis in other basins, with different synoptic environments, should also be considered.

\section{Acknowledgements}

The present work is part of the first author's thesis work at the Université Paul Sabatier Toulouse III, France. Based on a French initiative, AMMA was built by an international scientific group and is currently funded by a large number of agencies, especially from France, UK, US and Africa. It has been the beneficiary of a major financial contribution from the European Community's Sixth Framework Research Programme. Detailed information on scientific coordination and funding is available on the AMMA International web site http://www.amma-international.org. Meteosat-9 raw data were provided by Mr. Jean-Pierre Olry from SATMOS (CNRS/INSU and Météo-France). We thank Dr. Ron McTaggart-Cowan for helpful comments on a former version, and the three anonymous reviewers for their constructive suggestions.

\section{References}

Agusti-Panareda, A., Vasiljevic, D., Beljaars, A., Bock, O., Guichard, F., Nuret, M., Garcia Mendez, A., Anderson, E., Bechtold, P., Fink, A., Hersbach, H., Lafore, J.-P., Ngamini, J.-B., Parker, D.J., Redelsperger, J.-L., Tompkins, A., 2009. Radiosonde humidity bias correction over the West African region for the special AMMA reanalysis at ECMWF. Quart. J. Roy. Meteor. Soc. $135,595-617$.

Aiyyer, A.R., Thorncroft, C., 2006. Climatology of vertical wind shear over the tropical Atlantic. J. Climate 19, 2969-2983.

Arnault, J., Roux, F., 2009. Case-study of a developing African easterly wave during NAMMA: an energetic point of view. J. Atmos. Sci. 66, 2991-3020.

Arnault, J., Roux, F., 2010. Failed cyclogenetic evolution of a West-African monsoon perturbation observed during AMMA SOP-3. J. Atmos. Sci. 67, 1863-1883.

Avila, L.A., Clark, G.B., 1989. Atlantic tropical systems of 1988. Mon. Wea. Rev. $117,2260-2265$

Berry, G., Thorncroft, C., 2005. Case study of an intense African easterly wave. Mon. Wea. Rev. 133, 752-766.

Berry, G., Thorncroft, C., Hewson, T., 2006. African easterly waves during 2004 - analysis using objective techniques. Mon. Wea. Rev. 135, 1251-1267.

Beven II, J.L., Avila, L.A., Blake, E.S., Brown, D.P., Franklin, J.L., Knabb, R.D., Pasch, R.J., Rhome, J.R., Stewart, S.R., 2008. Atlantic hurricane season of 2005. Mon. Wea. Rev. 136, 1109-1173.

Bister, M., Emanuel, K.A., 1997. The genesis of Hurricane Guillermo: TEXMEX analyses and a modelling study. Mon. Wea. Rev. 125, 2662-2682.

Braun, S.A., 2010. Reevaluating the rôle of the Saharan Air Layer in Atlantic tropical cyclogenesis and evolution. Mon. Wea. Rev. 138, 2007-2037.

Brennan, M.J., Knabb, R.D., Mainelli, M., Kimbrelain, T.B., 2009. Atlantic hurricane season of 2005. Mon. Wea. Rev. 136, 1109-1173.

Brown, D.P., Beven, J.L., Franklin, J.L., Blake, E.S., 2010. Atlantic hurricane season of 2008. Mon. Wea. Rev. 138, 1975-2001.

Burpee, R.W., 1974. Characteristics of North African easterly waves during the summers of 1968 and 1969. J. Atmos. Sci. 31, 1556-1570.

Carlson, T.B., 1969a. Synoptic histories of three African disturbances that developed into Atlantic hurricanes. Mon. Wea. Rev. 97, 256-288.

Carlson, T.B., 1969b. Some remarks on African disturbances and their progress over the tropical Atlantic. Mon. Wea. Rev. 97, 716-726.

Carlson, T.B., Prospero, J.M., 1972. The large-scale movement of Saharan air outbreaks over the northern equatorial Atlantic. J. Appl. Meteor. 11, 283-297.

Davis, C.A., Trier, S.B., 2007. Mesoscale convective vortices observed during BAMEX. Part I: kinematic and thermodynamic structure. Mon. Wea. Rev. 135, 2029-2049.

Dunion, J.P., Velden, C.S., 2004. The impact of the Saharan Air Layer on Atlantic tropical cyclone activity. Bull. Amer. Meteor. Soc. 85, 353-365.

Fink, A.H., Vincent, D.G., Reiner, P.M., Speth, P., 2004. Mean state and wave disturbances during Phases I, II and III of GATE based on ERA-40. Mon. Wea. Rev. 132, 1661-1683.

Franklin, J.L., Brown, D.P., 2008. Atlantic hurricane season of 2006. Mon. Wea. Rev. 136, 1174-1200.

Franklin, J.L., Pasch, R.J., Avila, L.A., Beven II, J.L., Lawrence, M.B., Stewart, S.R., Blake, E.S., 2006. Atlantic hurricane season of 2004. Mon. Wea. Rev. 134, 981-1025.

Fritsch, J.M., Murphy, J.D., Kain, J.S., 1994. Warm core vortex amplification over land. J. Atmos. Sci. 51, 1780-1807.

Gray, W.M., 1968. Global view of the origin of tropical disturbances and storms. Mon. Wea. Rev. 96, 669-700.

Hendricks, E.A., Montgomery, M.T., Davis, C.A., 2004. On the role of "vortical" hot towers in tropical cyclone formation. J. Atmos. Sci. 61, 1209-1232. 
Jenkins, G.S., Pratt, A.S., Heymsfield, A., 2008. Possible linkages between Saharan dust and tropical cyclone rain band invigoration in the eastern Atlantic during NAMMA-06. Geophys. Res. Lett. 35, L08815.

Karyampudi, V.M., Carlson, T.N., 1988. Analysis and numerical simulations of the Saharan air layer and its effect on easterly wave disturbances. J. Atmos. Sci. 45, 3102-3136.

Karyampudi, V.M., Palm, S.P., Reagen, J.A., Fang, H., Grant, W.B., Hoff, R.M., Moulin, C., Pierce, H.F., Torres, O., Browell, E.V., Melfi, S.H., 1999 Validation of the Saharan dust plume conceptual model using Lidar, Meteosat, and ECMWF data. Bull. Amer. Meteor. Soc. 80, 3102-3136.

Kerns, B., Greene, K., Zipser, E., 2008. Four years of tropical ERA-40 vorticity maxima tracks. Part I: climatology and vertical vorticity structure. Mon. Wea. Rev. 136, 4301-4319.

Landsea, C.W., 2000. Climate Variability of Tropical Cyclones: Past, Present and Future. In: Pielke Sr., R.A., Pielke Jr., R.A. (Eds.), Storms. Routledge, New York, pp. 220-241.

Lavaysse, C., Flamant, C., Janicot, S., Knippertz, P., 2010. Links between African easterly waves, midlatitude circulation and intraseasonal pulsations of the West African heat low. Quart. J. Roy. Meteor. Soc. 136, 141-158.

Lin, Y.-L., Robertson, K.E., Hill, C.M., 2005. Origin and propagation associated with an African easterly wave as a precursor of hurricane Alberto (2000). Mon. Wea. Rev. 133, 3276-3298.

McBride, J.L., Zehr, R., 1981. Observational analysis of tropical cyclone formation, part II: comparison of non-developing versus developing systems. J. Atmos. Sci. 38, 1152-1166.

Montgomery, M.T., Nicholls, M.E., Cram, T.A., Saunders, A.B., 2006. A vortical hot tower route to tropical cyclogenesis. J. Atmos. Sci. 66, 355-386.

Pytharoulis, I., Thorncroft, C., 1998. The low-level structure of African easterly waves in 1995. Mon. Wea. Rev. 127, 2266-2280.

Raymond, D.J., Lopez-Carillo, C., Cavazos, L.L., 1998. Case-studies of developing east Pacific easterly waves. Quart. J. Roy. Meteor. Soc. 124, 2005-2034.

Redelsperger, J.-L., Thorncroft, C.D., Diedhiou, A., Lebel, T., Parker, D., Polcher, J., 2006. African monsoon, multidisciplinary analysis (AMMA): an international research project and field campaign. Bull. Amer. Meteor. Soc. 87, 1739-1746.

Reed, R.J., Norquist, D.C., Recker, E.E., 1977. The structure and properties of African wave disturbances as observed during phase III of GATE. Mon. Wea. Rev. 105, 317-333.
Riehl, H., 1954. Tropical Meteorology. Mc Graw Hill, New York, N.Y., USA. 392 pp.

Ritchie, E.A., Holland, G.J., 1997. Scale interactions during the formation of Typhoon Irving. Mon. Wea. Rev. 125, 1377-1396.

Roca, R., Lafore, J.-P., Piriou, C., Redelsperger, J.-L., 2005. Extra-tropical dry intrusions into the West African monsoon midtroposphere: an important factor for the convective activity over Sahel. J. Atmos. Sci. 62, 390-407.

Rodgers, R.F., Fritsch, J.M., 2001. Surface cyclogenesis from convectively driven amplification of midlevel mesoscale convective vortices. Mon. Wea. Rev. 129, 605-637.

Ross, R.S., Krishnamurti, T.N., 2007. Low-level African easterly wave activity and its relation to Atlantic tropical cyclogenesis in 2001. Mon. Wea. Rev. 135, 3950-3964.

Ross, R.S., Krishnamurti, T.N., Pattnaik, S., Simon, A., 2009. Energy transformation and diabatic processes in developing and non-developing African easterly waves observed during the NAMMA project of 2006. Wea. Forecasting 24, 1524-1538.

Sall, S.M., Sauvageot, H., Gaye, A., Viltard, A., de Félice, P., 2006. A cyclogenesis index for Tropical Atlantic off the African Coast. Atmos. Res. 79, 123-147.

Schwendike, J., Jones, S.C., 2010. Convection in an African easterly Wave over West Africa and the eastern Atlantic: a model case study of Helene (2006). Quart. J. Roy. Meteor. Soc. 136, 364-396.

Simpson, J., Ritchie, E., Holland, G.J., Halverson, J., Stewart, S., 1997. Mesoscale interactions in tropical cyclone genesis. Mon. Wea. Rev. 125, 2643-2661.

Thorncroft, C.D., Hodges, K., 2001. African easterly wave variability and its relationship to Atlantic tropical cyclone activity. J. Climate 14, 1166-1179.

Van Sang, N., Smith, R.K., Montgomery, M.T., 2008. Tropical-cyclone intensification and predictability in three dimensions. Quart. J. Roy. Meteor. Soc. 134, 563-582.

Wang, Z., Montgomery, M.T., Dunkerton, T.J., 2009. A dynamically-based method for forecasting tropical cyclogenesis location in the Atlantic sector using global model products. Geophys. Res. Lett. 36, L03801.

Zawislak, J., Zipser, E.J., 2010. Observation of seven African easterly waves in the East Atlantic during 2006. J. Atmos. Sci. 67, 26-43.

Zipser, E.J., et al., 2009. The Saharan Air Layer and the fate of African easterly waves. Bull. Amer. Meteor. Soc. 90, 1137-1156. 